\title{
Air Fluorescence Relevant for Cosmic-Ray Detection - Summary of the 5th Fluorescence Workshop, El Escorial 2007
}

\author{
Fernando Arqueros ${ }^{\mathrm{a}}$, Jörg R. Hörandel ${ }^{\mathrm{b}}$, Bianca Keilhauer ${ }^{\mathrm{c}}$ \\ ${ }^{a}$ Facultad de Ciencias Físicas, Universidad Complutense de Madrid, E-28040 Madrid, Spain \\ ${ }^{\mathrm{b}}$ Radboud Universiteit Nijmegen, Department of Astrophysics, P.O. Box 9010, 6500 GL Nijmegen, The Netherlands \\ ${ }^{\mathrm{c}}$ Universität Karlsruhe, Institut für Experimentelle Kernphysik, Postfach 3640, 76021 Karlsruhe, Germany
}

\begin{abstract}
High-energy cosmic rays with energies exceeding $10^{17} \mathrm{eV}$ are frequently observed by measurements of the fluorescence light induced by air showers. A major contribution to the systematic uncertainties of the absolute energy scale of such experiments is the insufficient knowledge of the fluorescence light yield of electrons in air. The aim of the 5th Fluorescence Workshop was to bring together experimental and theoretical expertise to discuss the latest progress on the investigations of the fluorescence light yield. The results of the workshop will be reviewed as well as the present status of knowledge in this field. Emphasis is given to the fluorescence light yield important for air shower observations and its dependence on atmospheric parameters, like pressure, temperature, and humidity. The effects of the latest results on the light observed from air showers will be discussed.
\end{abstract}

Key words: fluorescence yield, air showers PACS: 32.50.+d, 33.50.Dq, 87.64.kv, 96.50.sd

\section{Introduction}

The Earth is permanently exposed to a vast flux of particles from outer space. Most of these particles are fully ionized atomic nuclei, covering a large range in energy from the $\mathrm{MeV}$ regime to energies above $10^{20} \mathrm{eV}$. In 1962 the first event with an energy exceeding $10^{20} \mathrm{eV}$ was recorded [1]. Such cosmic rays are the highest-energy particles in the Universe, carrying the (macroscopic) energy of about $50 \mathrm{~J}$ concentrated on a single nucleus. Since their first discovery more than 40 years ago their origin has been an open question. "How do cosmic accelerators work and what are they accelerating?" is one of eleven science questions for the new century asked by the National Research Council of the National Academies of the United States [2], underlining the importance of this topic to astroparticle physics.
The properties of cosmic rays at highest energies are investigated with various experiments [3][4][5][6]. The flux of cosmic rays with energies exceeding $10^{20} \mathrm{eV}$ is below 1 particle per square kilometer and century. Thus, a reasonable measurement of these particles requires huge detectors operated stably over long periods of time. At present, this can be realized only with ground based experiments, registering the secondary particles generated by high-energy cosmic rays in the atmosphere. When a high-energy cosmic ray enters the Earth's atmosphere it induces a cascade of secondary particles, an extensive air shower. By far the most abundant particles in air showers are photons, electrons, and positrons, comprising the electromagnetic shower component. On their way through the atmosphere the (relativistic) charged particles emit Cherenkov radiation and excite nitrogen molecules to emit fluorescence light. A small fraction of the secondary 
particles eventually reaches the observation level. Over time, several methods to measure extensive air showers have been established. They can be divided into two groups: experiments measuring secondary particles (electrons, muons, and hadrons) reaching ground level and detectors observing the emitted Cherenkov or fluorescence photons. The latter allow for a three-dimensional reconstruction of the shower profile in the atmosphere.

A critical issue for all experiments is to establish an absolute energy scale for the measured showers. For experiments registering secondary particles at ground level this usually involves the usage of simulations of the shower development in the atmosphere, thus, introducing systematic uncertainties due to our limited knowledge of the hadronic interactions at such high energies.

On the other hand, fluorescence measurements of air showers provide a calorimetric measurement of the energy deposited in the air, being (nearly) independent of air shower simulations. The deposited energy is assumed to be proportional to the energy of the primary, shower inducing, particle. At present, this is the most direct and model independent method to determine the energy of an air shower. The main systematic uncertainty of this method arises from the insufficient knowledge of the fluorescence light yield of electrons in air. However, this is a quantity which can be measured in laboratory experiments, injecting electrons into air targets. Responsible for the fluorescence emission of the nitrogen molecules is mainly the electromagnetic shower component. The critical energy of electrons in air is about $84 \mathrm{MeV}$, thus, the bulk of particles has energies easily accessible at accelerators, or, at lower energies, even through radioactive $\beta$-decays.

This is the main focus of the present article. It gives an overview on the actual status of the knowledge of the fluorescence light yield of electrons in air, important for air shower detection. It summarizes the results of the 5th Fluorescence Workshop, which was held in El Escorial, Spain from September 16 th to 20th, 2007. After a short overview on the principle of the detection of air showers using fluorescence light (Sect.2.1), experiments applying this technique to register cosmic-ray induced air showers are described (Sect.2.2). The main physical processes involved in the production of fluorescence light in air are reviewed in Sect. 3. Contemporary experimental tools and theoretical treatments are discussed in Sect. 4. Latest data are compiled in Sect. 5, paying special attention to the dependence of the fluorescence light yield on atmospheric parameters, such as pressure, temperature, and humidity. In the following section (Sect.6) the influence of the results obtained on the light yield in air showers developing in realistic atmospheres is discussed. An outlook describing the next steps in determining the fluorescence yield in air concludes the article (Sect. 7). The most important pioneering measurements of the fluorescence light yield are summarized in an accompanying article [7].

\section{The fluorescence technique}

\subsection{Principle of air shower detection with fluorescence light}

A high-energy cosmic ray entering the atmosphere induces a cascade of secondary particles. One way to determine the energy of the primary particle is to measure the energy deposited in an absorber (i.e. the atmosphere), this is called a calorimetric energy measurement (e.g. [8]). If the shower is absorbed completely, the energy of the primary particle is identical to the energy deposited. However, in an air shower some energy is escaping a calorimetric measurement: a fraction of secondary particles reaches ground level and some energy is carried away by "invisible" particles such as neutrinos. Luckily, corrections for this effect are small and rather model independent, as will be discussed below. The calorimetric measurement by means of fluorescence light detection uses the fact that secondary particles in showers (mostly electrons and positrons) deposit energy in the atmosphere by ionization or excitation of air molecules. The excited nitrogen molecules subsequently relax to their ground state partially by the emission of fluorescence photons. The light is emitted isotropically, which implies that showers can be viewed from the side, thus, telescopes can observe large fiducial volumes of air. Most of the fluorescence light is emitted in the near UV region with wavelengths between about 300 and $400 \mathrm{~nm}$. Simulation studies show that most of the energy deposited into the atmosphere arises from electrons (and positrons) with energies below $1 \mathrm{GeV}$ with a maximum at about $30 \mathrm{MeV}[9]$. It is commonly assumed that the number of emitted fluorescence photons is proportional to the energy deposited in the atmosphere.

The number of fluorescence photons $\mathrm{d} N_{\gamma}$ which are generated in a layer of atmosphere with thick- 
ness $\mathrm{d} X$ registered by a fluorescence detector can be expressed as ${ }^{1}$

$$
\frac{\mathrm{d} N_{\gamma}}{\mathrm{d} X}=\int \frac{\mathrm{d}^{2} N_{\gamma}^{0}}{\mathrm{~d} X \mathrm{~d} \lambda} \cdot \tau_{a t m}(\lambda, X) \cdot \varepsilon_{F D}(\lambda) \mathrm{d} \lambda .
$$

$\varepsilon_{F D}$ denotes the efficiency of the fluorescence detector and $\tau_{a t m}$ the transmission of the atmosphere. The latter includes all transmission losses due to optical absorption, Rayleigh scattering, and Mie scattering from the point of emission to the detector. The number of emitted fluorescence photons $\mathrm{d} N_{\gamma}^{0}$ emitted per wavelength interval $\mathrm{d} \lambda$ and matter traversed $\mathrm{d} X$ is obtained as

$$
\begin{array}{r}
\frac{\mathrm{d}^{2} N_{\gamma}^{0}}{\mathrm{~d} X \mathrm{~d} \lambda}=\int Y(\lambda, P, T, u, E) \cdot \frac{\mathrm{d} N_{e}(X)}{\mathrm{d} E} \\
\cdot \frac{\mathrm{d} E_{d e p}}{\mathrm{~d} X} \mathrm{~d} E .
\end{array}
$$

The energy spectrum of the electrons (and positrons) at an atmospheric depth $X$ is given by $\mathrm{d} N_{e}(X) / \mathrm{d} E$ and $\mathrm{d} E_{d e p} / \mathrm{d} X$ describes the energy deposited in a layer of atmosphere with thickness $\mathrm{d} X$. The fluorescence light yield $Y$ describes the number of emitted photons per deposited energy (photons per MeV). For a calorimetric measurement we are interested in the deposited energy, thus, this definition relates the searched quantity directly to the observed amount of light. In the literature $Y$ is frequently given in units of photons per meter (or photons per unit length). This definition has the disadvantage that the number of photons emitted per unit length changes with varying air density. It is non-trivial to convert the two quantities into each other. Throughout this article we use the definition of photons per deposited energy, unless noted otherwise.

The fluorescence light yield $Y$ at a wavelength $\lambda$ depends on the atmospheric pressure $P$, the temperature $T$, the humidity $u$, and, in principle, as well on the energy of the electrons $E$. If the light yield is assumed to be energy independent, it can be taken out of the integral yielding

$$
\frac{\mathrm{d}^{2} N_{\gamma}^{0}}{\mathrm{~d} X \mathrm{~d} \lambda}=Y(\lambda, P, T, u) \cdot \frac{\mathrm{d} E_{d e p}^{t o t}}{\mathrm{~d} X} .
$$

The total energy deposited in an atmospheric layer with thickness $\mathrm{d} X$ is written as

$$
\frac{\mathrm{d} E_{d e p}^{t o t}}{\mathrm{~d} X}=\int \frac{\mathrm{d} N_{e}(X)}{\mathrm{d} E} \cdot \frac{\mathrm{d} E_{d e p}}{\mathrm{~d} X} \mathrm{~d} E .
$$

\footnotetext{
1 In contrast to later on, we use in this section the fluorescence yield per unit wavelength interval.
}

With (1) the relation

$$
\begin{array}{r}
\frac{\mathrm{d} N_{\gamma}}{\mathrm{d} X}=\frac{d E_{d e p}^{t o t}}{\mathrm{~d} X} \int Y(\lambda, P, T, u) \cdot \tau_{a t m}(\lambda, X) \\
\cdot \varepsilon_{F D}(\lambda) \mathrm{d} \lambda
\end{array}
$$

is obtained. It shows that the number of fluorescence photons detected is proportional to the energy deposited in the atmosphere. It remains to be shown that the fluorescence yield is indeed independent of the electron energy, see Sect. 4.2.2 below.

To calculate the energy of the primary particle from the observed fluorescence light still some corrections are necessary. The observed light contains also a contamination of Cherenkov light, either direct light (mostly emitted in forward direction) or scattered light. This effect has to be corrected for on an event-to-event basis [10]. It has to be taken into account that the cascade is not absorbed completely in the atmosphere and secondary particles reach ground level (longitudinal leakage of the calorimeter). Furthermore, particles which are not detected (neutrinos, high-energy muons) carry away energy. This "invisible" energy depends slightly on the mass of the primary particle and the hadronic interaction model used to describe the shower development in the atmosphere. Investigations of the Auger Collaboration indicate that the correction factor varies between 1.07 and 1.17 only, assuming primary protons or iron nuclei and applying different hadronic interaction models [11].

For illustration, we discuss here the systematic uncertainties of the absolute energy scale of the Pierre Auger Observatory (as evaluated before the 5th Fluorescence Workshop) [12]. The fluorescence telescopes are end-to-end calibrated using a large homogeneous light source which leaves an uncertainty of $9.5 \%$. Uncertainties in the shower reconstruction contribute with $10 \%$ and the correction of the invisible energy adds another $4 \%$ to the error budget. The atmospheric profile above the observatory is regularly monitored [13]. However, an uncertainty of about $4 \%$ for the energy scale of an individual event remains. In addition, uncertainties related to the fluorescence yield have to be taken into account. The dominant contribution is due to the absolute light yield (14\%). The dependence on atmospheric parameters contributes with $7 \%$. This results in a total systematic uncertainty of $22 \%$. Values for other experiments are similar and confirm that the biggest uncertainty for the absolute energy scale is the insufficient knowledge of the flu- 
orescence yield $Y(\lambda, p, T, u)$. To review the latest progress on the determination of this value is the objective of the present article.

\subsection{Air shower experiments applying the fluorescence technique}

In the following, the principle set-ups of air shower detectors applying the fluorescence technique are briefly sketched. Illustratively, a fluorescence detector for such an experiment has to be able to observe a $100 \mathrm{~W}$ light bulb ${ }^{2}$ moving at the speed of light through the atmosphere watched from a distance of $30 \mathrm{~km}$. To realize this, large-aperture telescopes are used to focus the light on cameras equipped with fast photomultiplier tubes, sensitive in the near UV region. ${ }^{3}$ First ideas to use the Earth's atmosphere as vast scintillation detector were discussed in the early 1960s [14]. The early history of experiments applying the fluorescence technique is summarized elsewhere [15].

\subsubsection{Initial experiments}

The pilot experiment to study the feasibility of detecting air showers with the fluorescence technique was the "Cornell Wide Angle System" proposed and built by K. Greisen and colleagues in the 1960s [16]. It consisted of three detector stations set up in the vicinity of the Cornell University campus. Each station comprised five photomultiplier tubes in a hexahedron arrangement, with a tube pointing north, south, east, west, and upward, respectively. The four radial tubes were tilted upwards by $30^{\circ}$. The system was operational for about 1000 hours. Light flashes were recorded, but they could not be attributed to air showers beyond doubt.

In 1967, a full scale fluorescence experiment was constructed by Greisen's group. It comprised 500 photomultiplier tubes, each corresponding to a pixel with a solid angle of $0.01 \mathrm{sr}$. The photomultipliers were divided into ten modules, each of them was equipped with a $0.1 \mathrm{~m}^{2}$ Fresnel lens. The experiment was operated for several years but was not sensitive enough to detect high-energy cosmic rays.

Similar activities were conducted by the Tokyo group leading to the INS-Tokyo experiment. In 1969 they recorded first clear fluorescence light signals

\footnotetext{
2 The fluorescence light of a $10^{17} \mathrm{eV}$ shower corresponds to a light bulb of about $100 \mathrm{~W}$.

3 Recent results of air shower experiments applying the fluorescence technique are summarized in e.g. [3][4][5][6].
}

from an extensive air shower with an energy exceeding $5 \cdot 10^{18} \mathrm{eV}[17]$.

\subsubsection{The Fly's Eye experiment}

In 1976 physicists from the University of Utah detected fluorescence light from cosmic-ray air showers. Three prototype modules were used at the site of the Volcano Ranch air shower array near Albuquerque, New Mexico. Each module comprised a $1.8 \mathrm{~m}$ diameter mirror for light collection with a camera consisting of 14 photomultiplier tubes at the focal plane. Fluorescence light was recorded in coincidence with an air shower array. These prototypes led eventually to the development of the Fly's Eye detector.

The Fly's Eye observatory [18] consisted of two stations, separated by $3.3 \mathrm{~km}$. The first one (Fly's Eye 1) comprised 67 front aluminized spherical section mirrors, with a diameter of $157 \mathrm{~cm}$. Winston light collectors and photomultipliers were hexagonally packed in groups of either 12 or 14 light sensing units, or "eyes" mounted in the focal plane of each mirror. The photomultipliers (EMI 9861B) had a fairly uniform quantum efficiency over the spectral range from 310 to $440 \mathrm{~nm}$. A motorized shutter system kept the "eyes" both light tight and weather proof during the day and permitted exposure to the sky at night. Each mirror unit was housed in a single, motorized corrugated steel pipe about $2.13 \mathrm{~m}$ long and $2.44 \mathrm{~m}$ in diameter. The units were turned down with mirror and open end facing the ground during the day and turned up at night to a predetermined position so that each "eye" observed an angular region of the sky. In total, 880 "eyes" were observing the complete upper hemisphere. The projection of each hexagonal "eye" onto the celestial sphere resembles the compound "eye" of an insect, hence, the name Fly's Eye. The second telescope (Fly's Eye II) was a smaller array of identical units, with 120 "eyes" in total, observing roughly one azimuthal quadrant of the night sky with elevation angles ranging between $2^{\circ}$ and $38^{\circ}$ above the horizon. Whenever the first telescope recorded an event, it sent an infrared flash of light towards the second telescope, which recorded pulse integrals and arrival times. The shower track geometry was reconstructed either from hit patterns and timing information by a single Fly's Eye detector or by stereoscopic viewing and relative timing by both Fly's Eyes. The experiment has been operated between 1981 and 1993. For the first time the fluorescence technique has been 
applied successfully to explore the properties of ultra high-energy cosmic rays on a large scale.

\subsubsection{The HiRes experiment}

The High Resolution Fly's Eye experiment (HiRes) was located in Utah, USA $\left(40^{\circ} \mathrm{N}, 112^{\circ} \mathrm{W}\right)$ [19]. It was the successor of the Fly's Eye experiment. HiRes consisted of two detector sites (Hires I \& II) separated by $12.6 \mathrm{~km}$, providing almost $360^{\circ}$ azimuthal coverage, each. Both telescopes were formed by an array of detector units. The mirrors consisted of four segments and formed a $5.1 \mathrm{~m}^{2}$ spherical mirror. At its focal plane an array of $16 \times 16$ photomultiplier tubes was situated, viewing a solid angle of $16^{\circ} \times 16^{\circ}$. HiRes I consisted of 22 detectors, arranged in a single ring, overlooking between $3^{\circ}$ and $17^{\circ}$ in elevation. This detector used an integrating $\mathrm{ADC}$ read-out system, which recorded the photomultiplier tubes' pulse height and time information. HiRes II comprised 42 detectors, set up in two rings, looking between $3^{\circ}$ and $31^{\circ}$ in elevation. It was equipped with a $10 \mathrm{MHz}$ flash $\mathrm{ADC}$ system, recording pulse height and timing information from its phototubes. The experiment has been operated between 1997 and 2007.

\subsubsection{Telescope Array}

The Telescope Array is an air shower experiment in the West Desert of Utah (USA), 140 miles south of Salt Lake City $\left(39.3^{\circ} \mathrm{N}, 112.9^{\circ} \mathrm{W}\right)[20]$. It comprises 576 scintillator stations and three fluorescence detector sites on a triangle with about $35 \mathrm{~km}$ separation. Each fluorescence detector station is equipped with 12 to 14 telescopes, viewing $3^{\circ}-33^{\circ}$ in elevation and $\approx 108^{\circ}$ in azimuth [21]. The telescopes have a segmented spherical mirror with a diameter of $3.3 \mathrm{~m}$ and a focal length of $3.0 \mathrm{~m}$. Each telescope has a camera comprising 256 photomultiplier tubes (Hamamatsu R9508), corresponding to a pixel size of about $1^{\circ}$ each. The sensitive area of a camera is $1 \mathrm{~m} \times 1 \mathrm{~m}$, which corresponds to a field of view of $15^{\circ}$ in elevation and $18^{\circ}$ in azimuth. The photomultipliers are read out by a FADC system. The experiment has been taking data since 2007 .

\subsubsection{The Pierre Auger Observatory}

The observatory combines the observation of fluorescence light with imaging telescopes and the measurement of particles reaching ground level in a "hybrid approach" [22]. The southern site (near Malargüe, Argentina, 35.2 ${ }^{\circ} \mathrm{S}, 69.5^{\circ} \mathrm{W}, 1400 \mathrm{~m}$ above sea level) of the worlds largest air shower detector consists of 1600 water Cherenkov detectors set up in an area covering $3000 \mathrm{~km}^{2}$. Four telescope systems overlook the surface array. A single telescope system comprises six telescopes, overlooking separate volumes of air. Each telescope is situated in a bay, protected by a remotely operated shutter. Light enters the bay through an UV transmitting filter and a ring of corrector lenses. A circular diaphragm (2.2 $\mathrm{m}$ diameter), positioned at the center of curvature of a spherical mirror, defines the aperture of the Schmidt optical system. A $3.5 \mathrm{~m} \times 3.5 \mathrm{~m}$ spherical mirror focuses the light onto a camera with an array of $22 \times 20$ hexagonal pixels. Each pixel is a photomultiplier tube, complemented by light collectors. Each camera pixel has a field of view of approximately $1.5^{\circ}$ in diameter. A camera overlooks a total field of view of $30^{\circ}$ azimuth $\times 28.6^{\circ}$ elevation. The photomultiplier signals are read out by FADC systems. The observatory has been completed in 2008 and has been taking data stably with a growing number of detector stations since 2004 .

Presently (2008), the Pierre Auger Collaboration is extending the observatory to lower energies. For this objective additional high-elevation telescopes (HEAT, High Elevation Auger Telescopes) are being built, covering an angular range from $30^{\circ}$ to $60^{\circ}$ in elevation [23]. These telescopes will be operated with an additional infill array of surface detectors combined with underground muon counters (AMIGA, Auger Muons and Infill for the Ground Array) [24] and antennae to detect radio emission from air showers [25]. To complete and extend the investigations begun in the South, the Pierre Auger Collaboration presently prepares an observatory in the northern hemisphere in Colorado, USA [26]. The set-up will, similarly to the southern site, comprise water Cherenkov detectors and fluorescence telescope systems.

\subsubsection{ASHRA}

The All-sky Survey High Resolution Air-shower telescope (ASHRA) is a proposed detector system to simultaneously measure Cherenkov and fluorescence light on the entire sky with 1 arc min resolution [27]. It is planned to install two stations at a distance of about 30 to $40 \mathrm{~km}$ on an island in Hawaii. A station comprises 12 wide-angle telescopes. Each telescope has a field of view of $50^{\circ} \times 50^{\circ}$, read out by CMOS sensor arrays. 


\subsubsection{JEM-EUSO}

JEM-EUSO is a proposed super-wide field UV telescope to detect ultra high-energy cosmic rays with energies above $10^{20} \mathrm{eV}$ [28]. It will be attached to the International Space Station (ISS) and will observe fluorescence photons emitted by air showers from an orbit of about $430 \mathrm{~km}$ altitude. The three dimensional development of the shower is reconstructed from a series of images of the shower. The spatial resolution is about $0.75 \times 0.75 \mathrm{~km}^{2}$. A double Fresnel lens module with $2.5 \mathrm{~m}$ diameter is the baseline optics for the JEM-EUSO telescope. The focal surface is equipped with about 6000 multianode photomultipliers. The launch is planned for 2012.

\section{Physical processes involved in the generation of air-fluorescence light}

Electrons passing through the atmosphere deposit energy due to inelastic collisions with air molecules. A small fraction of them give rise to the production of the fluorescence light observed in the spectral range of interest $(290-430 \mathrm{~nm})$. This air-fluorescence light is produced by nitrogen molecules.

In this section the main features of the excitation and de-excitation of $\mathrm{N}_{2}$ molecules will be reviewed (Sect.3.1). Fluorescence quenching including the humidity effect will also be discussed (Sect.3.2). Finally, the definition of the various parameters associated with the fluorescence yield as used in the literature will be presented (Sect. 3.3).

\subsection{Electron excitation and radiative de-excitation}

A scheme of the molecular levels of $\mathrm{N}_{2}$ and $\mathrm{N}_{2}^{+}$is shown in Fig. 1. As is well known from elementary molecular physics, each electronic state is split in vibrational levels $v$. In addition, each vibrational level is split in rotational sub-levels following a complicated structure. Electron collision excites molecular nitrogen in the ground state to upper levels. Down going arrows in Fig. 1 represent the de-excitation processes giving rise to fluorescence radiation. Although transitions take place between individual rotational levels of the upper and lower states, the corresponding rotational structure of the molecular spectrum is not resolved in our experiments. Under moderate spectral resolution, transitions between vibrational levels give rise to molecular bands $v-v^{\prime}$

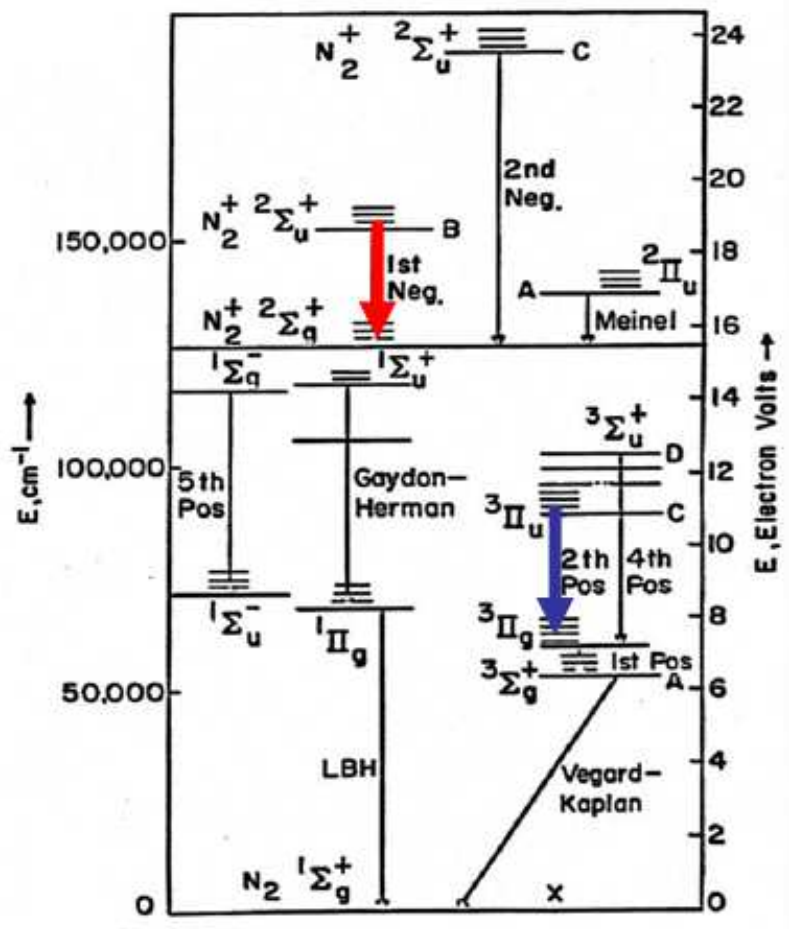

Fig. 1. Molecular levels of $\mathrm{N}_{2}$ and $\mathrm{N}_{2}^{+}$. Broad arrows represent the main transitions ( $1 \mathrm{~N}$ and $2 \mathrm{P}$ systems) [16].

with a spectral width and shape determined by the rotational structure ${ }^{4}$. The set of bands connecting a given pair of electronic states is named a band system.

In our spectral range, nitrogen fluorescence comes basically from the Second Positive system $\mathrm{C}^{3} \Pi_{u} \rightarrow$ $\mathrm{B}^{3} \Pi_{g}$ of $\mathrm{N}_{2}$ and the First Negative system $\mathrm{B}^{2} \Sigma_{u}^{+} \rightarrow$ $\mathrm{X}^{2} \Sigma_{g}^{+}$of $\mathrm{N}_{2}^{+}$(see Fig. 1) which in the air-fluorescence community are usually denoted as $2 \mathrm{P}$ and $1 \mathrm{~N}$ systems, respectively. Notice that while the $2 \mathrm{P}$ system is generated after the $\mathrm{N}_{2} \mathrm{X}^{1} \Sigma_{g}^{+} \rightarrow \mathrm{C}^{3} \Pi_{u}$ excitation, $1 \mathrm{~N}$ fluorescence takes place as a consequence of the $\mathrm{X}^{1} \Sigma_{g}^{+} \rightarrow\left(\mathrm{N}_{2}^{+}\right) \mathrm{B}^{2} \Sigma_{u}^{+}$molecular ionization, leaving the nitrogen ion in a specific excited state. The wavelengths of the molecular bands of nitrogen are well known (see for instance [30]).

Apart from the $1 \mathrm{~N}$ and $2 \mathrm{P}$ systems the weak bands of the $\mathrm{N}_{2}$ Gaydon-Herman system $(\mathrm{GH})$ have been observed in the air-fluorescence spectrum [31][29].

A spectrum typically observed at high pressure between 280 and $430 \mathrm{~nm}$ for air is depicted in Fig. 2 [32]. The labels mark 21 major transitions. All important transitions and the corresponding wave-

\footnotetext{
$\overline{4 \text { See e.g. }}[29]$ for some illustrative examples.
} 


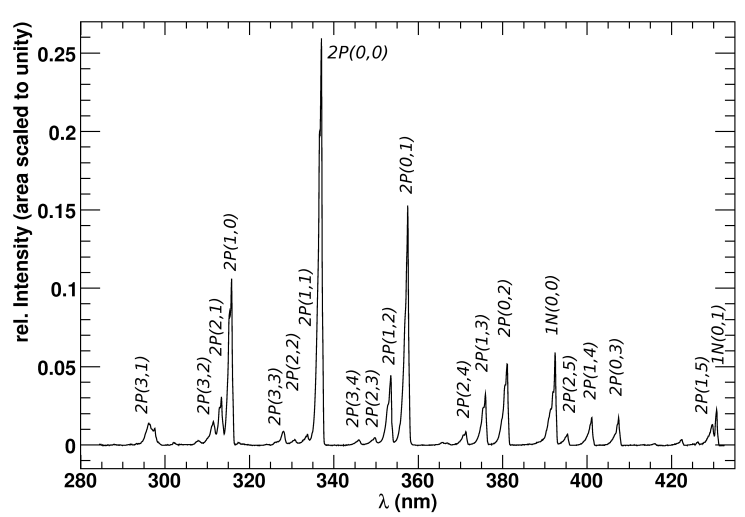

Fig. 2. Air fluorescence spectrum excited by $3 \mathrm{MeV}$ electrons at $800 \mathrm{hPa}$ as measured by the AIRFLY Collaboration [32].

Table 1

Transitions and corresponding wavelengths of the airfluorescence spectrum [32].

\begin{tabular}{ccccc}
\hline transition & $\lambda(\mathrm{nm})$ & & transition & $\lambda(\mathrm{nm})$ \\
\cline { 5 - 5 } $2 \mathrm{P}(3,1)$ & 296.2 & & $\mathrm{GH}(0,5)$ & 366.1 \\
$2 \mathrm{P}(2,0)$ & 297.7 & & $\mathrm{P}(3,5)$ & 367.2 \\
$\mathrm{GH}(6,2)$ & 302.0 & $2 \mathrm{P}(2,4)$ & 371.1 \\
$\mathrm{GH}(5,2)$ & 308.0 & $2 \mathrm{P}(1,3)$ & 375.6 \\
$2 \mathrm{P}(3,2)$ & 311.7 & $2 \mathrm{P}(0,2)$ & 380.5 \\
$2 \mathrm{P}(2,1)$ & 313.6 & $2 \mathrm{P}(4,7)$ & 385.8 \\
$2 \mathrm{P}(1,0)$ & 315.9 & $\mathrm{GH}(0,6)$ & 387.7 \\
$\mathrm{GH}(6,3)$ & 317.6 & $1 \mathrm{~N}(1,1)$ & 388.5 \\
$2 \mathrm{P}(4,4)$ & 326.8 & $1 \mathrm{~N}(0,0)$ & 391.4 \\
$2 \mathrm{P}(3,3)$ & 328.5 & $2 \mathrm{P}(2,5)$ & 394.3 \\
$2 \mathrm{P}(2,2)$ & 330.9 & $2 \mathrm{P}(1,4)$ & 399.8 \\
$2 \mathrm{P}(1,1)$ & 333.9 & $2 \mathrm{P}(0,3)$ & 405.0 \\
$2 \mathrm{P}(0,0)$ & 337.1 & $2 \mathrm{P}(3,7)$ & 414.1 \\
$\mathrm{GH}(0,4)$ & 346.3 & $2 \mathrm{P}(2,6)$ & 420.0 \\
$2 \mathrm{P}(2,3)$ & 350.0 & $1 \mathrm{~N}(1,2)$ & 423.6 \\
$2 \mathrm{P}(1,2)$ & 353.7 & $2 \mathrm{P}(1,5)$ & 427.0 \\
$2 \mathrm{P}(0,1)$ & 357.7 & $1 \mathrm{~N}(0,1)$ & 427.8 \\
\hline
\end{tabular}

lengths between 290 and $430 \mathrm{~nm}$ are compiled in Tab. 1.

The cross section for excitation of the upper electronic levels of both systems as a function of electron energy is displayed in Fig. 3. The curve for the $2 \mathrm{P}$ system shows a sharp maximum at about $15 \mathrm{eV}$ followed by a fast $E^{-2}$ decrease, as expected from the optically forbidden nature of this transition. On the contrary, the excitation cross section for the $1 \mathrm{~N}$ system shows a much softer maximum at about 100

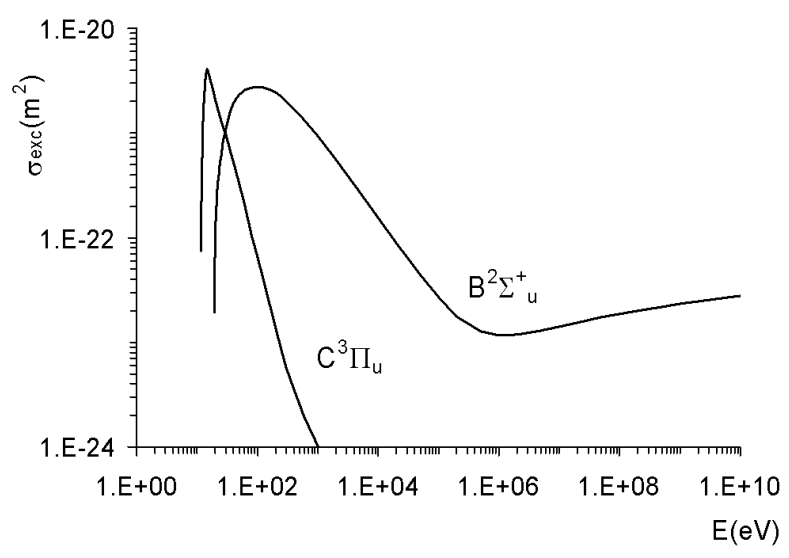

Fig. 3. Total cross sections for the excitation of the electronic states $\mathrm{C}^{3} \Pi_{u}$ and $\mathrm{B}^{2} \Sigma_{u}^{+}$versus electron energy [34].

$\mathrm{eV}$ followed by a much slower $(\log E) / E$ decrease which becomes a soft growing behavior at relativistic energies [33][34].

For a given electronic state the cross section for the excitation to a vibrational level $v$ is proportional to the Franck-Condon factor $q_{X \rightarrow v}$, defined as the overlapping integrals between the vibrational wave functions of the lower and upper levels of the excitation process. The Einstein coefficients $A_{v v^{\prime}}$ give the probability per unit time of radiative de-excitation $v-v^{\prime}$. Therefore, the probability of emission of a fluorescence $v-v^{\prime}$ photon by electron impact is proportional to the optical cross section defined as

$$
\sigma_{v v^{\prime}}=\sigma_{v} \frac{A_{v v^{\prime}}}{\sum_{v^{\prime}} A_{v v^{\prime}}}=\sigma_{v} B^{v v^{\prime}},
$$

and therefore, in the absence of other effects, the relative intensity of a molecular band with respect to a reference transition (e.g. 0-0) of the same system is given by

$$
\frac{I_{v v^{\prime}}^{0}}{I_{00}^{0}}=\frac{\sigma_{v v^{\prime}}}{\sigma_{00}}=\frac{q_{X \rightarrow v}}{q_{X \rightarrow 0}} \frac{B^{v v^{\prime}}}{B^{00}} .
$$

Tabulated values for both parameters $q_{X \rightarrow v}$ and $A_{v v^{\prime}}$ are available in the literature [35][36]. The relative intensities between bands of different systems can also be predicted using the relative values of the corresponding excitation cross sections. Transition probabilities determine the radiative lifetime $\tau^{r}$ of the excited level

$$
\frac{1}{\tau_{v}^{r}}=A_{v}=\sum_{v^{\prime}} A_{v v^{\prime}} .
$$

Notice that, as shown below, in a laboratory experiment both relative intensities (7) and lifetime 
(8) have to be corrected by the effect of collisional quenching.

\subsection{Fluorescence quenching}

At high pressure, molecular de-excitation by collision with other molecules of the medium plays an important role (collisional quenching). At a given temperature, the corresponding transition probability $A_{c}^{v}$ is proportional to the collision frequency and, thus, to the gas pressure $P$. The characteristic pressure $P_{v}^{\prime}$ is defined as the one for which the probability of collisional quenching equals that of radiative de-excitation $A_{v}^{c}\left(P_{v}^{\prime}\right)=A_{v}$,

$$
A_{v}^{c}(P)=A_{v} \frac{P}{P_{v}^{\prime}} .
$$

Thus, the fluorescence intensity in the absence of quenching $I_{v v^{\prime}}^{0}$ is reduced by the Stern-Volmer factor [37]

$$
I_{v v^{\prime}}(P)=I_{v v^{\prime}}^{0} \frac{1}{1+P / P_{v}^{\prime}}
$$

From (7) and (10) the relative intensities of molecular bands at high pressure $\left(P \gg P^{\prime}\right)$ become ${ }^{5}$

$$
\frac{I_{v v^{\prime}}}{I_{00}}=\frac{q_{X \rightarrow v}}{q_{X \rightarrow 0}} \frac{B^{v v^{\prime}}}{B^{00}} \frac{P_{v}^{\prime}}{P_{0}^{\prime}} .
$$

Collisional quenching enlarges the total transition probability and, therefore, the lifetime of the population of excited molecules $\tau_{v}$ is shortened as compared with the radiative one as

$$
\frac{1}{\tau_{v}}=\frac{1}{\tau_{v}^{r}}+\frac{1}{\tau_{v}^{c}}
$$

with $\tau_{v}^{c}=1 / A_{v}^{c}$. As a result, the effective lifetime decreases with pressure as

$$
\frac{1}{\tau_{v}(P)}=\frac{1}{\tau_{v}^{r}}\left(1+\frac{P}{P_{v}^{\prime}}\right) \text {. }
$$

Both $\tau_{v}^{r}$ and $P_{v}^{\prime}$ can be measured in a plot of reciprocal lifetime versus pressure (Stern-Volmer plot). This is a very well established technique in use since many years for the experimental determination of radiative lifetimes and quenching cross sections. In principle, a measure of the fluorescence intensity versus pressure (10) also provides a determination of $P_{v}^{\prime}$. However, as discussed in detail later, in a laboratory experiment the above relationship might be

\footnotetext{
5 Experimental confirmation of a collisional mechanism populating vibrational levels of the $\mathrm{C}^{3} \Pi_{u}$ state which might induce an additional pressure dependence of the $2 \mathrm{P}$ relative intensities has been shown in [38][39].
}

distorted because of the effect of secondary electrons leading to systematic uncertainties in the measurement of the characteristic pressures.

The probability of collisional de-excitation per unit time of a molecule in a given upper level $v$ can be expressed ${ }^{6}$ in terms of the quenching rate constant $K_{Q}$ as $A_{c}=N K_{Q}$ where $N$ is the number of molecules per unit volume. In the case of pure nitrogen

$$
K_{Q}=\sigma_{\mathrm{NN}} \bar{v}
$$

and

$$
\bar{v}=\sqrt{\frac{16 k T}{\pi M}},
$$

where $\sigma_{\mathrm{NN}}$ is the cross section for collisional deexcitation between nitrogen molecules, $\bar{v}$ is the mean value of the relative velocity of molecules in the gas, $T$ is the absolute temperature, $M$ is the molecular nitrogen mass, and $k$ is the Boltzmann's constant. Since $P=N k T$, the characteristic pressure for pure nitrogen can be expressed as

$$
P_{\mathrm{N}}^{\prime}=\frac{k T}{\tau} \frac{1}{\sigma_{\mathrm{NN}} \bar{v}}=\frac{\sqrt{\pi M k T}}{4 \sigma_{\mathrm{NN}}} \frac{1}{\tau^{r}} .
$$

The above expressions can be generalized for a mixture of gases as

$$
\frac{1}{P^{\prime}}=\sum_{i} \frac{f_{i}}{P_{i}^{\prime}}
$$

where $f_{i}$ is the fraction of molecules of type $i$ in the mixture and

$$
P_{i}^{\prime}=\frac{k T}{\tau} \frac{1}{\sigma_{\mathrm{N} i} \bar{v}_{\mathrm{N} i}} .
$$

In the general case, the relative velocity $\bar{v}_{\mathrm{N} i}$ is given by [40]

$$
\bar{v}_{\mathrm{N} i}=\sqrt{\frac{8 k T}{\pi \mu}},
$$

where $\mu=M_{\mathrm{N}} M_{i} /\left(M_{\mathrm{N}}+M_{i}\right)$ is the reduced mass of the two body system $\mathrm{N}-i$.

For dry air the above sum includes basically nitrogen and oxygen with $f_{\mathrm{N}}=0.79$ and $f_{\mathrm{O}}=0.21$. However, in practice, air contains also other components. For instance, the effect of argon can be treated by (17) and (18) accordingly.

A particular interesting case is the effect of water vapor. The characteristic pressure of humid air $P_{h u m}^{\prime}$ containing a fraction $f_{\mathrm{H}_{2} \mathrm{O}}$ of water molecules, that

\footnotetext{
6 In the next paragraphs all collisional parameters will be assumed to correspond to a given molecular level $v$.
} 
is a water vapor pressure $P_{\mathrm{H}_{2} \mathrm{O}}=P f_{\mathrm{H}_{2} \mathrm{O}}$, is related with that of dry air $P_{d r y}^{\prime}$ by

$$
\frac{1}{P_{\text {hum }}^{\prime}}=\frac{1}{P_{d r y}^{\prime}}\left(1-\frac{P_{\mathrm{H}_{2} \mathrm{O}}}{P}\right)+\frac{P_{\mathrm{H}_{2} \mathrm{O}}}{P} \frac{1}{P_{\mathrm{H}_{2} \mathrm{O}}^{\prime}} \text {. }
$$

Laboratory measurements of $P^{\prime}$ for nitrogen with variable quantities of water vapor, argon, oxygen, etc. provide values of the corresponding $P_{i}^{\prime}$ pressures and, therefore, the dependence of fluorescence intensity on environmental conditions.

Quenching collision is a very complex problem of molecular physics and basically no reliable theoretical predictions on cross sections are available. Therefore, a description of the dependence of fluorescence quenching on pressure and humidity relies on experimentally determinated $P^{\prime}$ values.

Furthermore, fluorescence quenching depends on temperature for a given density and air composition. Firstly, the frequency of collisions increases with $\bar{v}$ and, thus, $P^{\prime}$ grows with $\sqrt{T}(16)$. Secondly, the collisional cross section is a function of the kinetic energy of the colliding particles and, thus, $\sigma_{\mathrm{N} i}$ is a function of $T$. While the first dependence obeys well known laws of the kinetic theory of gases, the second one is again associated to the molecular problem of collisional de-excitation, in this case on the dependence of the quenching cross section on collision energy. Very few experimental studies of the temperature dependence for nitrogen fluorescence are available. On the other hand, no simple theory has been developed capable to predict the temperature dependence of quenching. The collisional cross section is assumed to follow a power law in temperature $^{7}, \sigma \propto T^{\alpha}$, where the $\alpha$-parameter might be either positive or negative, depending on the nature of the partners and the type of interaction. The $\alpha$ coefficient might even be valid only for certain temperature ranges, since the dominating type of interaction varies with the velocity of the molecules. As a consequence, from (10) a dependence of fluorescence intensity as

$$
\frac{1}{I} \propto 1+b T^{\alpha-\frac{1}{2}}
$$

can be predicted for a temperature scan at constant pressure, while the dependence at constant gas density $\rho=P /\left(R_{\text {gas }} T\right)\left(R_{\text {gas }}\right.$ is the specific gas constant) follows

$$
\frac{1}{I} \propto 1+b^{\prime} T^{\alpha+\frac{1}{2}}
$$

\footnotetext{
7 See [41],$[42]$, and [43] for discussions on the $T$ dependence of the quenching cross section.
}

where $b$ and $b^{\prime}$ are constants.

In this volume new interesting measurements of the $T$ dependence for pure nitrogen [41] and air [44] will be presented.

\subsection{Fluorescence yield}

Several parameters can be used to quantify the intensity of air-fluorescence radiation in regard with the energy deposited by electrons. In addition, the same physical magnitudes are denominated in the literature with different names and/or using different symbols.

The main physical magnitudes are the following:

- Number of fluorescence photons emitted per unit electron path length. Several authors (for instance [34][45][46][47][48]) name it fluorescence (or photon) yield.

- Fraction of deposited energy emitted as fluorescence radiation (without units) named fluorescence efficiency in [45][46][47].

- Number of fluorescence photons emitted per unit deposited energy. For several authors (for instance [29][49][50]) this parameter is the fluorescence yield.

In this article we will use the following definitions and symbols:

$-\varepsilon_{\lambda}\left[\mathrm{m}^{-1}\right]$ is the number of fluorescence photons with wavelength $\lambda$ corresponding to a given transition $v-v^{\prime} 8$ per unit of electron path length.

$-\Phi_{\lambda}$ is the fluorescence efficiency, defined as the fraction of deposited energy emitted as fluorescence radiation.

- The fluorescence yield $Y_{\lambda}\left[\mathrm{MeV}^{-1}\right]$ is defined as the number of fluorescence photons emitted per unit deposited energy.

The ratio of $\Phi_{\lambda}$ and $Y_{\lambda}$ is easily given by the energy of the photons $E_{\lambda}=h \nu$ with $\nu=c / \lambda$. However, the relationship between $\varepsilon_{\lambda}$ and $Y_{\lambda}$ is not straightforward. As discussed below, fluorescence light is basically generated by secondary electrons produced in ionization processes. These secondaries have a non-negligible range and, therefore, measured fluorescence intensity depends on geometrical features of the observation volume. A precise measurement of the fluorescence yield requires the evaluation of

\footnotetext{
8 In the next paragraphs, until the end of this section, the wavelength $\lambda$ will characterize the molecular transition instead of the $v v^{\prime}$ pair. This is a more compact notation very common in articles of the air-fluorescence community.
} 
deposited energy in the same gas volume from where fluorescence is being detected.

The total number $\varepsilon_{\lambda}$ of fluorescence photons generated per unit path length in a very large medium can be expressed as a function of the optical cross section $\sigma_{\lambda}$ of the transition by [34]

$$
\varepsilon_{\lambda}=N \frac{\sigma_{\lambda}}{1+P / P_{\lambda}^{\prime}}
$$

where $N$ is the density of nitrogen molecules and $P_{\lambda}^{\prime}$ is the characteristic pressure of the upper level $v$ of the transition. Obviously, $\varepsilon_{\lambda}$ depends on electron energy because of the energy dependence of the optical cross section.

In a laboratory experiment with a finite observation volume, a fraction ${ }^{9}$ of these photons is not detected by the system. Equation (23) can be applied using an effective optical cross section $\sigma_{\lambda}^{\text {eff }}$ as defined in [33].

The number of fluorescence photons per unit column density per electron is given by

$$
\frac{\varepsilon_{\lambda}}{\rho}=\frac{N_{A}}{M_{g a s}} \frac{\sigma_{\lambda}^{e f f}}{1+P / P_{\lambda}^{\prime}}=\frac{A_{\lambda}}{1+P / P_{\lambda}^{\prime}},
$$

where $\rho$ is the gas density, $N_{A}$ is Avogadro's number and $M_{\text {gas }}$ is the mass of the gas molecules. The value of $\varepsilon_{\lambda} / \rho$ in the absence of quenching $(P=0)$ is named $A_{\lambda}$ in [45][46].

The fluorescence efficiency depends on pressure as

$$
\Phi_{\lambda}=\Phi_{\lambda}^{0} \frac{1}{1+P / P_{\lambda}^{\prime}} .
$$

At zero pressure, $\Phi_{\lambda}$ is given by

$$
\Phi_{\lambda}^{0}=\frac{\rho A_{\lambda} h \nu}{(\mathrm{d} E / \mathrm{d} X)_{d e p}},
$$

where $(\mathrm{d} E / \mathrm{d} X)_{\text {dep }}$ is the energy deposited per unit electron path length in the same volume where fluorescence photons have been generated (see below for more details on the effect of secondary electrons).

Finally, the fluorescence yield follows the same pressure dependence as $\Phi_{\lambda}$

$$
Y_{\lambda}=Y_{\lambda}^{0} \frac{1}{1+P / P_{\lambda}^{\prime}},
$$

and its value in the absence of quenching is given by

$$
Y_{\lambda}^{0}=\frac{\Phi_{\lambda}^{0}}{h \nu}
$$

\footnotetext{
9 This fraction depends on the gas pressure and the geometrical features of the experimental set-up.
}

Equations (27) and (28) can be written as [45][46]

$$
Y_{\lambda}=\frac{1}{(\mathrm{~d} E / \mathrm{d} X)_{d e p}} \frac{\rho A_{\lambda}}{1+\rho B_{\lambda} \sqrt{T}},
$$

where

$$
B_{\lambda}=\frac{R_{g a s} \sqrt{T}}{P_{\lambda}^{\prime}} .
$$

The dependence of the fluorescence yield on pressure, temperature, humidity, etc. can be predicted from either (27) and (28) or (29), using the characteristic pressure as given above in (17) and (18).

\section{The actual status}

This section describes the progress in the last few years on the experimental and theoretical tools developed for air-fluorescence studies. In Sect. 4.1 the modern experimental techniques used for the measurement of fluorescence yield and its dependence on atmospheric parameters are described. Electron sources, target features, detection systems as well as the various techniques developed for the absolute calibration of the systems are described. Finally, in Sect. 4.2 theoretical results on the processes leading to the emission of air fluorescence light and the relation to deposited energy are discussed.

\subsection{Experimental techniques}

Any experimental set-up consists basically of three components: a source of electrons (or $\alpha$ particles) properly monitored, a collision chamber where air or any gas mixture is excited by the electrons, and an optical as well as an electronic system to register the fluorescence light intensity.

\subsubsection{Electron sources}

Three types of sources are used in air-fluorescence experiments: electron beams from accelerators in large facilities, radioactive sources, and low-energy electron guns in laboratories.

4.1.1.1. Accelerators They can provide electron beams with a small diameter typically of about few millimeters. Different kinds of accelerators are available for the various energy ranges $(\mathrm{keV}-\mathrm{GeV})$. In particular, they are the only possible source for very high-energy electrons. A disadvantage of this technique is the large background signal induced in the fluorescence detectors which requires a careful subtraction from the fluorescence signal. Furthermore, 
electrons exit the accelerator line through a window of a certain thickness and material dependent on the energy range.

The FLASH Collaboration [48][49][51] used the Final Focus Test Beam facility at the Stanford Linear Accelerator Center which provided $28.5 \mathrm{GeV}$ electrons in 3 ps pulses of about $10^{8}$ electrons at a rate of $10 \mathrm{~Hz}$.

The MACFLY Collaboration [52] used the CERN/SPS-X5 test beam facility which delivers a pulsed electron beam of about $10^{4}$ electrons per spill (4.8 s duration) every $16.8 \mathrm{~s}$. Measurements at 20 and $50 \mathrm{GeV}$ were carried out using this facility.

The AIRFLY Collaboration exploits this technique in an ambitious program to measure the fluorescence yield in the interval $6 \mathrm{keV}-420 \mathrm{MeV}$ using four different accelerators [53]. The interval $6-30 \mathrm{keV}$ is covered by the Advanced Photon Source of the Argonne National Laboratory. The intense synchrotron x-ray beam of the 15-ID line of this accelerator produces an almost monochromatic beam of electrons through photoelectric and Compton interactions with the ambient air. Also at the Argonne National Laboratory, the Chemistry Division electron Van de Graaff accelerator operated in pulsed mode at $60 \mathrm{~Hz}$, with beam currents from 0.2 to $0.8 \mu \mathrm{A}$, was used by this collaboration to get electrons in the range $0.5-3 \mathrm{MeV}$. Measurements in the energy range from 3 to $15 \mathrm{MeV}$ were performed at the Argonne Wakefield Accelerator. The LINAC was operated at $5 \mathrm{~Hz}$, with bunches of maximum charge of $1 \mathrm{nC}$, length $15 \mathrm{ps}$ (FWHM), and a typical energy spread of $\pm 0.3 \mathrm{MeV}$ at $14 \mathrm{MeV}$. Finally, measurements in the energy region $50-420 \mathrm{MeV}$ were performed by AIRFLY at the Beam Test Facility of the INFN Laboratori Nazionali di Frascati, which can deliver electrons with intensity ranging from single particle up to about 100 particles per bunch at a repetition rate of $50 \mathrm{~Hz}$ with a typical pulse duration of $10 \mathrm{~ns}$.

4.1.1.2. Radioactive sources Beta emitters provide electrons with a continuous energy spectrum. In particular, ${ }^{90}{ }^{\mathrm{Sr}-}{ }^{90} \mathrm{Y}$ sources with a maximum and average energy of 2.3 and $0.85 \mathrm{MeV}$, respectively, are widely used. This energy range, around the minimum of the energy loss curve, is of great interest in air-fluorescence studies. In this technique fluorescence detection is based on electron-photon coincidences.

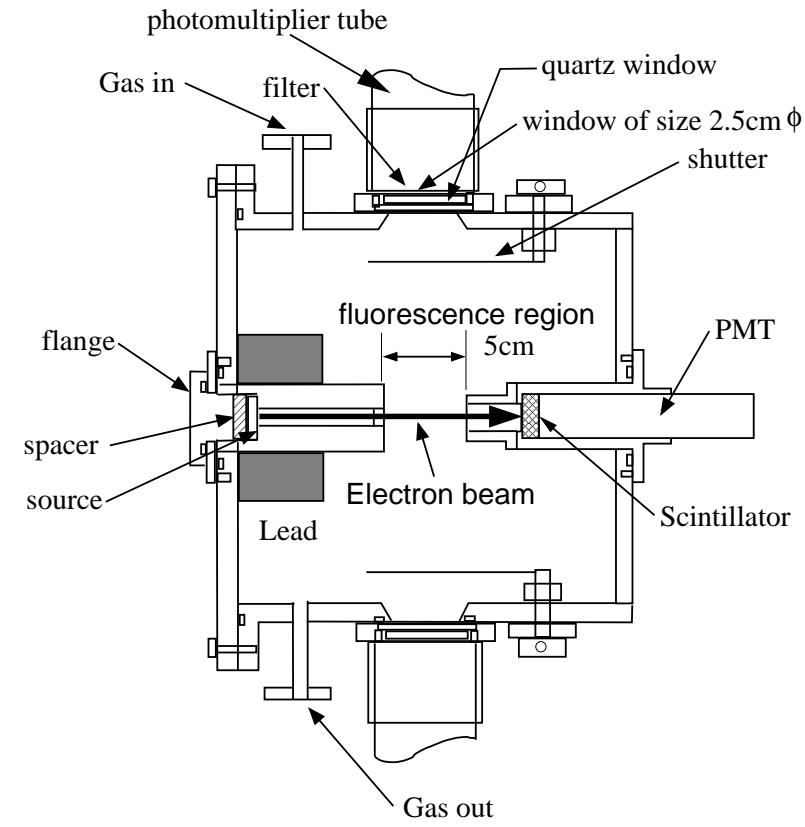

Fig. 4. Schematic drawing of the chamber (top view) used by Nagano et al. [46]. Three photomultipliers are mounted on two sides and the top of the chamber, they view fluorescence light through quartz windows. Optical filters are mounted between the photomultipliers and the windows. Electrons from a ${ }^{90} \mathrm{Sr}$ radioactive source are beamed and detected by a scintillation counter.

A schematic drawing of the chamber used by Nagano et al., applying this technique, is presented in Fig. 4 [46].

An advantage of using radioactive sources is that once the source is safely located in the experimental set-up, long lasting experiments can be carried out at very low maintenance costs. However, this technique has also some disadvantages. The main one is that unless strong radioactive sources are used, the rate of coincidences is very low and, thus, very large data acquisition times are needed to achieve sufficient statistics, then increasing systematic uncertainties.

In the last years, sources with increasing activity have been used. Nagano et al. [46][47], using a 3.7 $\mathrm{MBq}{ }^{90} \mathrm{Sr}$ source, were able to measure the absolute value and the pressure dependence of fluorescence for isolated bands using broadband $10 \mathrm{~nm}$ filters. A source of $37 \mathrm{MBq}$ has allowed Waldenmaier et al. [50] (AIRLIGHT experiment) accurate measurements of the very short nitrogen lifetimes in air at high pressure. The most active source ever used in this field is the one of Lefeuvre et al. [54] with an activity of $370 \mathrm{MBq}$ which has allowed to record the spectrum 
with a monochromator of $6 \mathrm{~nm}$ resolution. Finally, the MACFLY Collaboration also used a ${ }^{90} \mathrm{Sr}$ source for measurements of the absolute fluorescence yield at $1.5 \mathrm{MeV}$ [52].

Alpha emitters are also a very useful tool for fluorescence studies. As an example, an interesting study on pure nitrogen using a ${ }^{241} \mathrm{Am}$ source of $3.7 \mathrm{kBq}$ has been carried out [41]. Alpha particles lose energy by excitation and ionization in the gas. Although direct excitation of the $\mathrm{N}_{2} 2 \mathrm{P}$ system is forbidden, low-energy secondary electrons excite the $\mathrm{C}^{3} \Pi_{u}$ upper level (see [41] for more details). Many important properties of air fluorescence like its dependence on pressure, temperature, and humidity can be studied using this technique. Again, the main disadvantage is the low fluorescence intensity due to the limited source activity which in this case might be more important for legal restrictions of alpha sources.

4.1.1.3. Low-energy electron guns Air-fluorescence emission induced by low-energy electrons $(E<0.1$ $\mathrm{MeV}$ ) is of great interest. In the first place, a nonnegligible fraction of the energy deposited in the atmosphere by a cosmic-ray shower is delivered by low-energy electrons [9]. Furthermore, the assumption of proportionality between fluorescence intensity and deposited energy might not be fulfilled at low electron energies [55].

Customized electron guns are being used for this application. Morozov et al. [56] employ a modified electron gun designed for monochrome displays. The cathode is operated at high negative potential and the anode is connected to the ground. The gun delivers electrons of about $12 \mathrm{keV}$. Electron pulses of about 5 ns FWHM are performed by means of the control grid of the electron gun.

Rosado et al. [57] have designed a novel gun. The electron emission is based on a plasma produced by a pulsed nitrogen laser focused on the cathode, with up to $30 \mathrm{~Hz}$ repetition rate. The cathode, which is maintained at a negative potential by a high voltage power supply, accelerates electrons to kinetic energies up to $30 \mathrm{keV}$. Electron pulses of about $20 \mathrm{~ns}$ width and $40 \mathrm{~mA}$ peak intensity are achieved using this technique.

For fluorescence studies at high pressure induced by low-energy electrons, a very thin window has to be used to isolate the electron gun from the collision chamber. Morozov et al. use an ultra thin $(300 \mathrm{~nm})$ silicon nitride window which allows the passage of

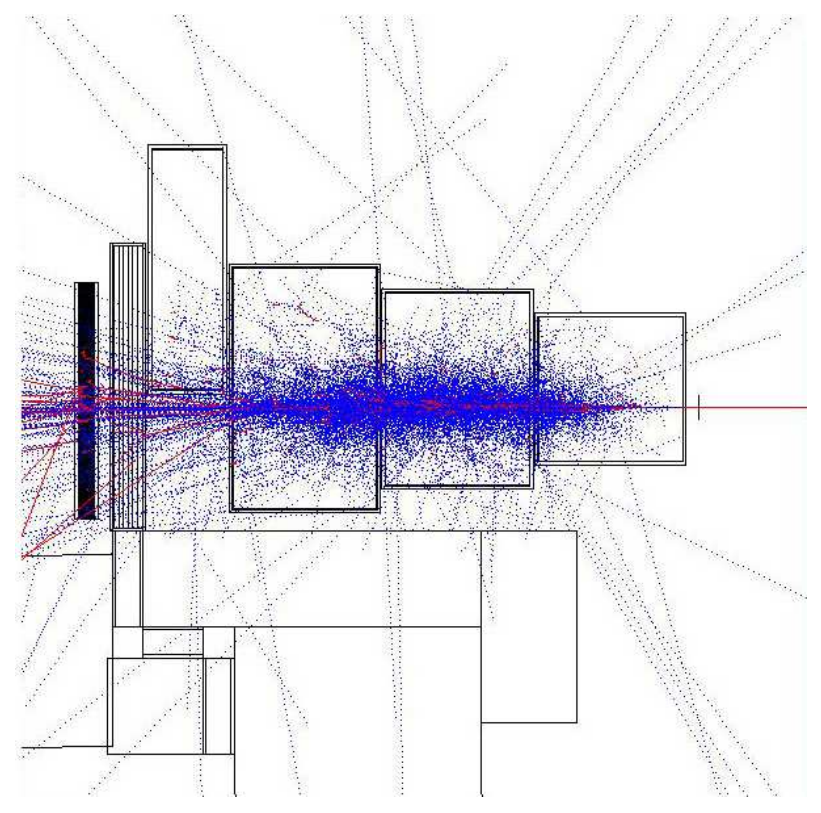

Fig. 5. Thick-target configuration of the FLASH Collaboration showing a GEANT3.2 simulation of an electromagnetic shower generated in the target [58][59].

$12 \mathrm{keV}$ electrons without substantial energy degradation. For the moment, Rosado et al. are working at low pressure. Differential pumping allows maintaining pressure in the electron gun well below $0.1 \mathrm{~Pa}$ to ensure cathode isolation, whereas working pressures up to $35 \mathrm{~Pa}$ can be used in the gas cell.

\subsubsection{The target}

Concerning the target two types of fluorescence experiments are being carried out. The so-called thick-target and thin-target experiments. For highenergy electrons $(E \gtrsim 1 \mathrm{MeV})$, air can be considered a thin target since the attenuation of the beam is very small even at atmospheric pressure. Most experiments described here are carried out under thin target conditions (e.g. the Nagano experiment shown in Fig. 4).

In our field thick-target experiments are those which use a dense medium where the high-energy electrons initiate an electromagnetic shower which enters the air collision chamber to produce fluorescence light. In other words, in these thick-target experiments the fluorescence light produced by an electromagnetic shower (created in a thick non-air target) on a thin air target is studied.

Two experiments have used the thick-target technique, FLASH and MACFLY. A schematic view of the thick-target configuration of the FLASH Col- 
laboration is displayed in Fig. 5. The result of a GEANT3.2 simulation of an electromagnetic shower generated in the target is shown [58][59]. The electron beam is incident on a variable-thickness ceramic alumina stack. This material has good thermal properties along with possessing a critical energy similar to that of air.

The MACFLY thick device [60] is composed of an internally black covered quasi cylindrical, large volume $\left(\approx 1 \mathrm{~m}^{3}\right)$, pressurized tank containing the gas under study. The electron beam, aligned with the axial symmetry of the chamber, is impinging on a pre-shower target. This variable thickness preshower system, is used to initiate electromagnetic showers inside the chamber.

A particular case is an air target at very low pressure $(<35 \mathrm{hPa})$ for the study of the fluorescence contribution of secondary electrons as used by Rosado et al. [57]. The experimental conditions, i.e. low pressure and low energy $(\sim 30 \mathrm{keV})$ are suitable for the analysis of spatial features of the fluorescence emission. This experiment has allowed to test the results of a model [55], discussed below in Sect.4.2.1, for the calculation of the fluorescence light generated by secondary electrons.

The accurate knowledge of the dependence of fluorescence intensity on environmental conditions is one of the most important goals in this field. The gas target where fluorescence is produced in the laboratory is in general a mixture of gases emulating air under various atmospheric conditions. Since airfluorescence light is basically produced by nitrogen, many experiments have been performed using pure nitrogen as target. Fluorescence in air is strongly quenched by oxygen collisions while pure nitrogen is much more efficient. Thus, properties of $\mathrm{N}_{2}$ fluorescence can be more easily studied using pure nitrogen.

Several experiments have been carried out to check the effect of argon on air fluorescence (e.g. [32]). The effect of humidity has been studied as well [44][49][50][56][61], adding to the mixture a known amount of water vapor. Another very important parameter is the air temperature. The dependence of fluorescence yield on temperature in a large interval, covering that found in the atmosphere, is an experimental challenge. Devices capable to provide air targets under controlled temperatures in the required interval have been designed by AIRFLY [44] (Fig. 6) and Fraga et al. [41]. See these articles for details on the chamber design.

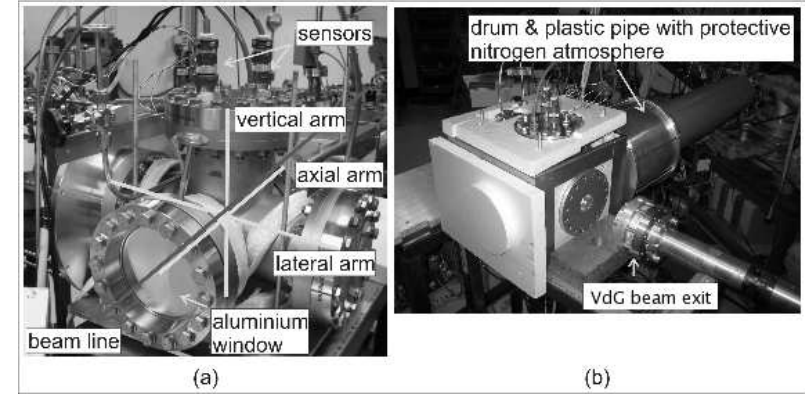

Fig. 6. The temperature chamber used by the AIRFLY Collaboration [44]: a) the chamber at the beam line as it appears before mounting the polystyrene box, b) the chamber inside the polystyrene box, with a protective drum and pipe also in place.

\subsubsection{Detection techniques}

The detection and analysis of the air-fluorescence radiation are carried out using the appropriate optical and electronic devices. In the first place, the emitted fluorescence light has to be collected and, if possible, spectroscopically analyzed. For the latter task, a set of filters or a monochromator are used. For interference filters the dependence of the spectral response on the incident angle has to be carefully measured [41][50][62]. If sufficient fluorescence intensity is available, a monochromator can be used to measure the fluorescence spectrum [32][54][57]. The spectrum provides a measure of the relative intensities which is a very valuable information. In addition, the dependence on pressure of either reciprocal lifetimes [50] or intensities [32][47] of spectroscopically resolved fluorescence yield allows to measure the $P_{v}^{\prime}$ values.

For the detection of fluorescence light, the most usual tool is a photomultiplier working in single photon counting regime. In fact, if possible, several photomultipliers viewing the collision chamber from several viewpoints allow a higher efficiency and the possibility to record simultaneously the fluorescence radiation in different spectral intervals. The AIRFLY Collaboration uses additionally a hybrid photodiode capable of single photoelectron counting [62].

\subsubsection{Absolute calibration}

The most important objective of this world-wide effort is an accurate measurement of the absolute value of the air-fluorescence yield. For this task, it is necessary to calibrate the detection system absolutely, including geometrical and transmission factors of the entire optical system. The absolute value of the number of electrons traversing the field of view 
of the collision chamber has to be measured.

To determine the fluorescence yield, the energy deposited by the electron beam inside the volume observed by the optical system has to be known. A first approach, which might be valid at low electron energy is to assume that the energy loss, as predicted by the Bethe-Bloch formula, equals the energy deposited by the electrons in the medium [45][46].

As discussed later, secondary electrons generated by the primary electron are mainly responsible of both the fluorescence emission and the energy deposition in the medium. Therefore, the total size of the volume where energy is deposited (and fluorescence is emitted) is related to the range of secondary electrons. For high-energy primaries, a non-negligible fraction of the energy is deposited by secondaries with a range larger than the typical size of the experimental collision volume observed by the optical system. In this case, a Monte Carlo simulation is very useful to determine the energy deposited in the interaction region accurately, including the geometrical features of the collision chamber and the optical field of view. Several standard Monte Carlo codes, like EGS4 [63] and GEANT4 [64] are being used for this purpose.

Several techniques have been developed for the absolute calibration of the optical systems. In principle, an accurate measurement of the geometrical features of the electron beam, the collection system, the transmission of all the optical elements, and the quantum efficiency of the light detector provide the necessary efficiency factor. This procedure has been applied by several experiments [47][50][52][54].

Other calibration procedures have been developed in order to reduce the (usually large) systematic uncertainties from the efficiency parameters mentioned above. These techniques rely on the comparison of fluorescence intensity with a well-known physical process leading to the emission of light with the same spectral and geometrical features. Two physical processes have been employed for this purpose. The first one uses the Cherenkov light emitted by the electron beam in the gas, while the second one is based on Rayleigh scattering from a laser beam replacing the electron beam.

The AIRFLY Collaboration [62] has developed the technique based on the comparison with Cherenkov light. The measurements are taken in two modes (Fig. 7). In the fluorescence mode, the isotropic fluorescence light produced by the electrons in the field of view of the detector is recorded. In this mode, contributions from other sources of
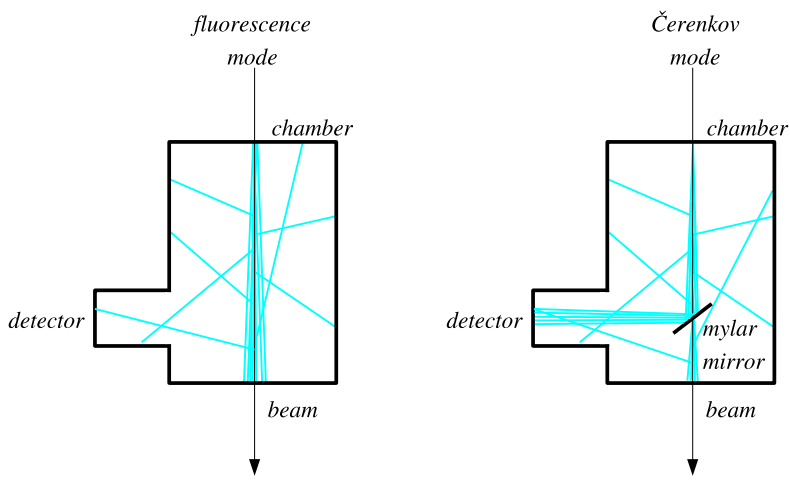

Fig. 7. Experimental set-up used by the AIRFLY Collaboration for the measurement of the absolute air-fluorescence yield [62].

light, like Cherenkov or transition radiation, are negligible due to the non-isotropic emission of such mechanisms. In the Cherenkov mode, a thin mylar mirror at an angle of $45^{\circ}$ is inserted remotely into the beam, redirecting the Cherenkov light into the detector. In this mode, the Cherenkov light fully dominates over fluorescence. The absolute fluorescence yield is then determined using the ratio of the signal measured in the fluorescence and in the Cherenkov configurations. The Cherenkov yield is known from theory, the geometrical factors of the apparatus are derived from a full GEANT4 simulation of the detector and take into account the probability of a photon being emitted in each case and also the fact that Cherenkov light is very directional and fluorescence light is emitted isotropically. Using this technique, the AIRFLY Collaboration has measured the absolute yield of the $337 \mathrm{~nm}$ band. A preliminary result has been presented already in [62].

The technique based on a comparison with the Rayleigh-scattered light was proposed by the FLASH and the Madrid groups at a previous workshop [65]. A nitrogen pulsed laser beam crosses the collision chamber in the place of the electron beam. Typical pulses of about $100 \mu \mathrm{J}$ energy and $4 \mathrm{~ns}$ width scatter a number of photons of the same order of magnitude as those from fluorescence runs. One of the main problems of this technique is light scattered at the walls of the chamber which has to be carefully suppressed. A measurement of the pressure dependence of the Rayleigh signal provides valuable information on the background scattered light and the linearity of the signal.

Using this technique, the FLASH Collaboration has already carried out a measurement of 
the absolute yield with an uncertainty below $10 \%$. The Madrid [57] and AIRLIGHT [50] groups are presently using this technique for the absolute calibration of their systems.

Two different strategies are being used for the measurement of the total fluorescence yield in the spectral interval of the telescopes. Several experiments [47][52][54] carry out an absolute measurement of the yield for the whole spectral interval including many molecular bands while [62] measures the absolute fluorescence yield of the main band $(337 \mathrm{~nm})$ and the contribution of the remaining spectral components is inferred later from an accurate measurement of the spectrum [32].

Notice that a comparison of results of the fluorescence yield in different spectral intervals needs a value for the relative intensities as well as the $P_{v}^{\prime}$ values, see (11).

\subsection{Theoretical approaches}

\subsubsection{Predictions on fluorescence emission}

The well-known physical processes leading to molecular excitation and fluorescence emission have been described in Sect. 3. Einstein coefficients, Franck-Condon factors as well as excitation and ionization cross sections are available in the literature, e.g. [35][36]. The amount of fluorescence photons generated by electrons traversing a given air thickness has been calculated in [33][34] using a Monte Carlo algorithm which takes into account the dominant role of secondary electrons. This algorithm also calculates the energy deposition for which secondary electrons are mainly responsible.

Bunner [16] realized early that secondary electrons from ionization processes are the main source of fluorescence light, since the excitation cross section of the corresponding upper levels (Fig. 3) shows a fast decrease with energy, in particular the one for the $2 \mathrm{P}$ system. Unfortunately, Bunner [16] was not able to calculate the fluorescence emission from secondary electrons since the necessary data, in particular, the spectrum of secondary electrons were not available at that time for collisions at high energy. An estimate of the energy spectrum of secondary electrons up to the $\mathrm{GeV}$ range has been used in [33][34] to calculate for the first time the fluorescence intensity induced by high-energy electrons. An improved energy spectrum of secondaries has allowed recently more reliable results of the fluorescence intensity and also a precise calculation of deposited

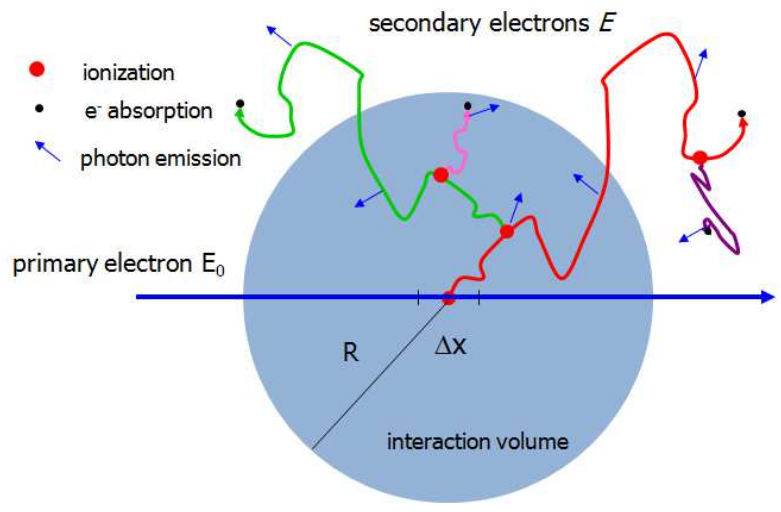

Fig. 8. The energy loss of a primary electron in $\Delta X$ gives rise to the production of secondaries, being mainly responsible for the fluorescence light emission. A fraction of both deposited energy and fluorescence emission might take place outside the observation region [55].

energy and, thus, of the fluorescence yield [55].

A schematic view of the processes involved in the emission of fluorescence light and the deposition of energy from secondary electrons as modeled in [55] can be seen in Fig. 8. A primary electron traversing an atmospheric depth $\Delta X$ may either excite or ionize a molecule. In the latter case, the secondary electron produces further excitations and/or ionizations until all secondaries are stopped in the medium. Both fluorescence generation and energy deposition due to molecular excitations/ionizations are calculated using a Monte Carlo algorithm. As a result, the energy deposited per unit path length of air as well as the number of molecules excited to the upper levels of the $2 \mathrm{P}$ and $1 \mathrm{~N}$ system are determined. The results for both magnitudes depend on the volume of the interaction region as well as the air pressure. In fact, it is a function of $P \times R$, where $R$ is the radius of the sphere around the interaction point defining the medium size. Neglecting the quenching effect, the ratio of both magnitudes gives $Y^{0}$, i.e. the fluorescence yield at $P=0$. Results on these predictions are compared with experimental data in [55].

The predicted values of the energy deposited per unit path length as a function of electron energy for several values of $P \times R$ are depicted in Fig. 9 [55]. As expected, deposited energy at very high $P \times R$ values tends to the total energy loss predicted by the Bethe-Bloch theory. Notice that for typical observation volumes in fluorescence experiments $\left(\approx 10^{3}\right.$ $\mathrm{hPa} \times \mathrm{cm})$, the deposited energy is smaller than the total energy loss by an amount which at atmospheric pressure ranges from about $18 \%$ at $1 \mathrm{MeV}$ to $42 \%$ at $10 \mathrm{GeV}$. 


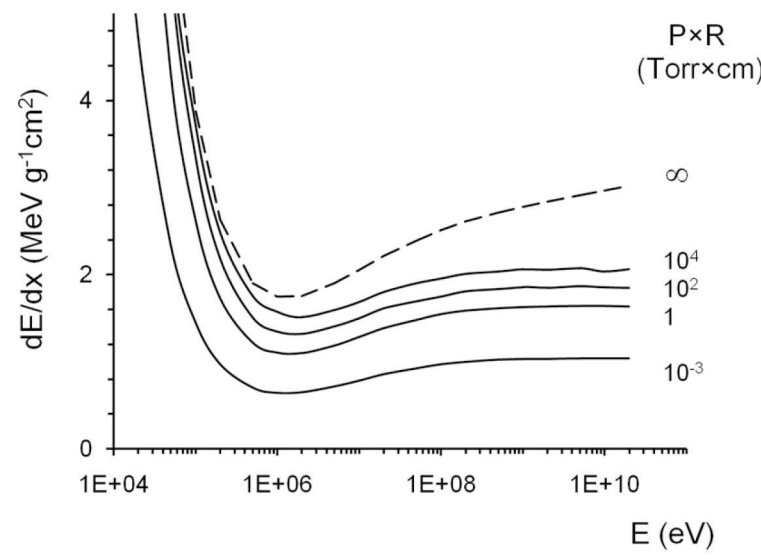

Fig. 9. Energy deposited by a primary electron per unit path length versus primary energy for several values of $P \times R$ (product of pressure times fiducial region, continuous lines). For very high $P \times R$ values deposited energy equals the energy loss of the primary electron predicted by the Bethe-Bloch theory (broken line) [55].

\subsubsection{The relation between fluorescence light intensity and deposited energy}

The fluorescence technique is based on the assumption that the fluorescence yield is independent of the electron energy. In other words, for given atmospheric conditions the fluorescence intensity is assumed to be proportional to the energy deposited. Therefore, the energy deposited by a shower at a given altitude is a function of atmospheric properties (pressure, temperature, humidity, etc.) but it is independent of the energy spectrum of the shower electrons. The validity of this assumption has been proven using both, theoretical and experimental tools. In the following, the theoretical results are summarized while experimental data are discussed later in Sect. 5.5.

From a theoretical point of view several arguments have been used in favor (with some caution) of an expected proportionality between fluorescence intensity and deposited energy. Ave et al. [53] argue that since the total number of secondary electrons produced by the passage of the primary electron in an air volume is roughly proportional to the energy deposited, the fluorescence light is also expected to be proportional to the energy deposited. On the other hand, Arqueros et al. [55] point out that the ratio of fluorescence emission and deposited energy is strongly dependent on the spectrum of lowenergy secondaries which in principle varies with the primary energy and the distance from the primary interaction. Thus, this proportionality has to be demonstrated with a detailed analysis.

The Monte Carlo algorithm in [55] is used for a detailed calculation of the energy dependence of the fluorescence yield. The results (see Fig. 6 - 9 in [55]) can be summarized as follows: the fluorescence yield decreases with primary energy about $10 \%$ in the range $1 \mathrm{keV}-1 \mathrm{MeV}$ and $4 \%$ in the interval $1 \mathrm{MeV}$ $-20 \mathrm{GeV}$ for the $337 \mathrm{~nm}$ band. For the $391 \mathrm{~nm}$ band the corresponding decrease is about $6 \%$ for the interval $1 \mathrm{keV}-1 \mathrm{MeV}$ and $1 \%$ for $1 \mathrm{MeV}-20 \mathrm{GeV}$. A smooth increase of the fluorescence yield with $P \times R$, smaller than $2 \%$ in the range $15-1500 \mathrm{hPa} \times \mathrm{cm}$, is found for energies larger than $1 \mathrm{MeV}$. At lower energy and/or region size the fluorescence yield is clearly not proportional to the deposited energy.

In summary, the theoretical results in [55] predict a very small dependence of the fluorescence yield on electron energy with no impact on the calibration of fluorescence telescopes.

\section{Compilation of data}

\subsection{Spectrum and pressure dependence}

As mentioned already, air-fluorescence emission in the range $290-430 \mathrm{~nm}$ is basically due to the $1 \mathrm{~N}$ and $2 \mathrm{P}$ systems of $\mathrm{N}_{2}$. The spectrum consists of molecular bands with a degraded shape. The wavelength and the intensity of these bands have been measured by many authors. In particular, their spectral positions are accurately known for many years, including the rotational structure. However, relative intensities depend strongly on the gas features (i.e. pressure, gas composition, etc.) as given by (11) and (17) - (19). Therefore, comparison between different authors is not straightforward.

Although data on the relative intensities of the air-fluorescence spectrum at several conditions have been published since long ago, well established results are not yet available. In the last years, the airfluorescence community has made a significant effort in achieving accurate values of the relative intensities and their pressure dependence.

Nagano et al. [47] have measured the relative intensities of 15 bands in both pure nitrogen and dry air. As already mentioned, in their experimental setup excitation is carried out with electrons from a $3.7 \mathrm{MBq}{ }^{90} \mathrm{Sr}$ radioactive source and fluorescence is spectroscopically resolved, using a set of interference filters. Relative intensities are measured by electronphoton coincidences. Values of the $P_{v}^{\prime}$ parameters 
Table 2

Experimental results of $P^{\prime}$ at $293 \mathrm{~K}$ for the $2 \mathrm{P}$ and $1 \mathrm{~N}$ system of nitrogen in air. The first column shows the vibrational number of the upper level of the transition with wavelength shown in the second column. See text for a brief description of the main experimental features. Uncertainties are not quoted. More details can be found in the original publications.

\begin{tabular}{|c|c|c|c|c|c|c|c|c|}
\hline \multicolumn{2}{|c|}{$\begin{array}{l}2 \mathrm{P} \text { system } \\
\mathrm{v} \lambda(\mathrm{nm})\end{array}$} & AIRFLY [29] & \multicolumn{2}{|c|}{ Nagano et al. $[47]^{\mathrm{a}}$} & Bunner $[16]^{\mathrm{b}}$ & $\begin{array}{l}\text { Pancheshnyi et al. [67][66] } \\
P^{\prime}(\mathrm{hPa})\end{array}$ & AIRLIGHT [50] ${ }^{\mathrm{d}}$ & MACFLY [52] \\
\hline \multirow{4}{*}{0} & 337.1 & 15.89 & 19.2 & \multirow{4}{*}{18.1} & \multirow{4}{*}{20.0} & \multirow{4}{*}{13.10} & \multirow{4}{*}{15.0} & \multirow{4}{*}{25.8} \\
\hline & 357.7 & 15.39 & 18.1 & & & & & \\
\hline & 380.5 & 16.51 & 19.4 & & & & & \\
\hline & 405.0 & 17.80 & 12.3 & & & & & \\
\hline \multirow{6}{*}{1} & 315.9 & 11.88 & 23 & \multirow{6}{*}{25.6} & \multirow{6}{*}{8.7} & \multirow{6}{*}{11.20} & \multirow{6}{*}{15.0} & \multirow{6}{*}{17.1} \\
\hline & 333.9 & 15.50 & - & & & & & \\
\hline & 353.7 & 12.70 & 30.6 & & & & & \\
\hline & 375.6 & 12.82 & 34.1 & & & & & \\
\hline & 399.8 & 13.60 & 24.2 & & & & & \\
\hline & 427.0 & 6.38 & 72 & & & & & \\
\hline \multirow{7}{*}{2} & 297.7 & 17.30 & - & \multirow{7}{*}{7.9} & \multirow{7}{*}{6.1} & \multirow{7}{*}{9.10} & \multirow{7}{*}{-} & \multirow{7}{*}{11.4} \\
\hline & 313.6 & 12.27 & - & & & & & \\
\hline & 330.9 & 16.90 & 40.2 & & & & & \\
\hline & 350.0 & 15.20 & - & & & & & \\
\hline & 371.1 & 14.80 & - & & & & & \\
\hline & 394.3 & 13.70 & 24.2 & & & & & \\
\hline & 420.0 & 13.80 & 7.3 & & & & & \\
\hline \multirow{3}{*}{3} & 296.2 & 18.50 & - & \multirow{3}{*}{-} & \multirow{3}{*}{3.3} & \multirow{3}{*}{7.90} & \multirow{3}{*}{-} & \multirow{3}{*}{8.8} \\
\hline & 311.7 & 18.70 & - & & & & & \\
\hline & 328.5 & 20.70 & - & & & & & \\
\hline 4 & 326.8 & 19.00 & - & - & - & - & - & - \\
\hline & 385.8 & 19.00 & - & & & & & \\
\hline
\end{tabular}

\begin{tabular}{llllccccc}
\hline 1N system & & & & & & \\
& 391.4 & 2.94 & 5.02 & 4.83 & 1.44 & 2.4 & 1.23 & 3.17 \\
0 & 427.8 & 2.89 & - & - & - & - & - & - \\
\hline 1 & 388.5 & 3.9 & - & - & - & - & - & - \\
\hline
\end{tabular}

a The second column of Nagano et al. values are weighted averages which are provided in their publication.

b Weighted averages.

${ }^{\mathrm{c}}$ Inferred from quenching rate constant and lifetime measurements at $337 \mathrm{~nm}(2 \mathrm{P} v=0), 316 \mathrm{~nm}(2 \mathrm{P} v=1), 314 \mathrm{~nm}(2 \mathrm{P}$ $v=2), 414 \mathrm{~nm}(2 \mathrm{P} v=3)$ and $391 \mathrm{~nm}(1 \mathrm{~N} v=0)$.

$\mathrm{d}$ Inferred from quenching rate constants and lifetime measurements at $337 \mathrm{~nm}(2 \mathrm{P} v=0), 316 \mathrm{~nm}(2 \mathrm{P} v=1), 391 \mathrm{~nm}(1 \mathrm{~N}$ $v=0)$.

are determined from measurements of the pressure dependence of fluorescence yield in the range $1-$ $10^{3} \mathrm{hPa}$ using (11). Results on relative intensities and characteristic pressures are given in Tabs. 1 and 2 of [47]. $P^{\prime}$ values are reviewed below in Tab. 2 for comparison with other authors. Notice that the determination of the $P_{v}^{\prime}$ values by Nagano et al. does not take into account the possible effect of secondary electrons escaping the field of view. Since this effect is pressure dependent, it might give rise to system- 


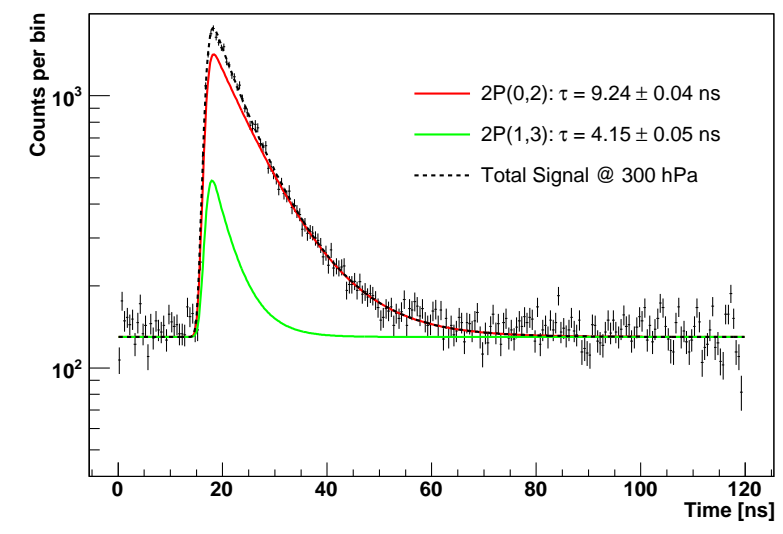

Fig. 10. Exponential time distribution between the electron signals in the scintillator and the photon signals measured by the AIRLIGHT experiment [50].

atic uncertainties.

The AIRLIGHT experiment [50], using a technique similar to that of Nagano et al. [46], have measured the relative intensities of 8 nitrogen bands. However, here the $P_{v}^{\prime}$ values are determined from the dependence of the fluorescence lifetime on pressure. Delayed electron - photon coincidences are detected to measure the exponential decay of the fluorescence emission, that is, the effective lifetime (Fig. 10).

As already mentioned, the reciprocal lifetime increases linearly with pressure (Stern-Volmer plot) and the corresponding slope provides the $P_{v}^{\prime}$ value as given by (13). Effective lifetimes at high pressure are very low (in the range of few nanoseconds) and, therefore, a high time resolution is necessary. This technique is free from possible systematic uncertainties due to secondary electrons escaping the field of view.

$P_{v}^{\prime}$ measurements in dry air, pure nitrogen, and several mixtures of nitrogen, oxygen, and water vapor allow Waldenmaier et al. [50] to determine quenching rate constants for the various components separately, see (16) - (18). The results shown in Tab. 2 of [50] can be used to calculate the $P_{v}^{\prime}$ values for any air-like mixture. As an example, the results for the $2 \mathrm{P}(\mathrm{v}=0,1)$ and $1 \mathrm{~N}(\mathrm{v}=0)$ transitions at $293 \mathrm{~K}$ are inferred from the corresponding quenching rates and lifetimes and are shown in Tab. 2 for comparison with other authors.

The AIRFLY Collaboration [32][29] has achieved very accurate results on the air-fluorescence spectrum using the electron beam of the Argonne Chemistry Van de Graaff facility. A high resolution Oriel MS257TM spectrograph combined with a $1024 \times$ 255 CCD pixel array (Andor DV420 BU2) allowed

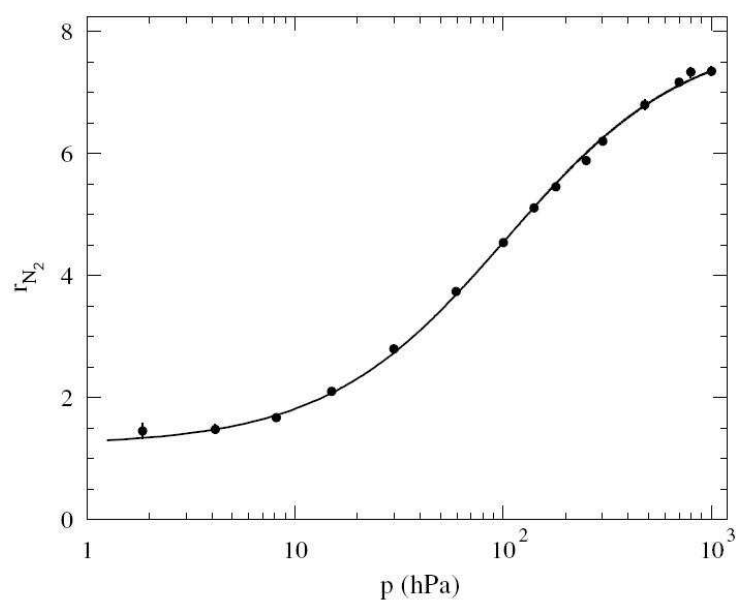

Fig. 11. The AIRFLY Collaboration determines the $\mathrm{P}^{\prime}{ }_{337}$ value for air from the ratio of nitrogen to air signals as a function of pressure [32].

to record high-resolution spectra of air fluorescence at $800 \mathrm{hPa}$ and $293 \mathrm{~K}$ (see Fig. 2). The spectral response was calibrated with an uncertainty of $3 \%$. Using this technique, 34 bands of nitrogen in the interval 280-429 $\mathrm{nm}$ including a few weak lines of the Gaydon-Herman system were identified. The measured relative intensities are listed in Tab. 1 in [32]. The experimental values for bands with a common upper level are in good agreement with the theoretical predictions given by the Einstein coefficients.

The measurements of the $P_{v}^{\prime}$ values were performed at the Argonne Wakefield Accelerator with $14 \mathrm{MeV}$ beam energy, operated in pulsed mode. The fluorescence intensity is recorded as function of pressure. However, the procedure followed here is different from the one of previous experiments e.g. [45][47]. AIRFLY compares the pressure dependence of the $337 \mathrm{~nm}$ band for nitrogen and dry air under the same experimental conditions (Fig. 11). Since the fraction of fluorescence losses due to secondary electrons escaping the field of view is basically the same for nitrogen and air, this comparison allows a determination of $P_{337}^{\prime}$ for quenching with $\mathrm{N}_{2}$ and $\mathrm{O}_{2}$ and, hence, for air. An accurate result of $P_{\text {air }}^{\prime}=15.89 \pm 0.73 \mathrm{hPa}$ is reported in [32][29]. The comparison (with respect to the $337 \mathrm{~nm}$ line) of the pressure dependence for other bands has provided a measure of $P_{v}^{\prime}$ for other spectral components of the air fluorescence light.

Following this technique, characteristic pressures of 25 bands of the $2 \mathrm{P}, 1 \mathrm{~N}$, and $\mathrm{GH}$ systems of nitrogen in air are reported in Tab. 2 of [32] with an 
uncertainty smaller than previous experiments. The results for the $2 \mathrm{P}$ and $1 \mathrm{~N}$ systems have been included in Tab. 2 for comparison with those of other authors.

The MACFLY experiment has measured the pressure dependence of the total (unresolved) fluorescence intensity $(290-440 \mathrm{~nm})$ for pure nitrogen and air (Figs. 5 and 6 in [52]). The standard theoretical formula ((4) in [52]) is fitted to the experimental pressure dependence, using previously reported values of the fluorescence yields at $P=0$ of pure nitrogen for 24 wavelengths, radiative lifetimes, and quenching rate constants at $T=0$ for $\mathrm{N}_{2}$ and $\mathrm{O}_{2}$. As a result, MACFLY reports dry-air $P_{v}^{\prime}$ values for five upper levels, $2 \mathrm{P}(v=0-3)$ and $1 \mathrm{~N}(v=0)$. Notice that these $P_{v}^{\prime}$ results are not obtained from direct measurements but are inferred as the most likely values, which are simultaneously consistent with the experimental pressure dependence of the spectrally unresolved fluorescence light yield and various experimental and theoretical data on relative intensities and quenching from other authors. The results are also shown in Tab. 2 for comparison.

Finally, the FLASH Collaboration reports the measurement of the air-fluorescence spectrum excited with electrons from the Final Focus Test Beam at $28.5 \mathrm{GeV}$ energy [49][51]. The spectrum was recorded using a spectrograph with about $1 \mathrm{~nm}$ resolution. Fig. 7 of [49] shows an illustrative example for air at $207 \mathrm{hPa}$. Relative intensities are compared in Fig. 10 of [49] with other experimental values. Measurements of both spectroscopically unresolved and resolved fluorescence intensity versus pressure were carried out by the FLASH Collaboration (see Tab. 1 and Fig. 8 in [49]).

As summary, most of the available results on characteristic pressures for the $2 \mathrm{P}$ and $1 \mathrm{~N}$ nitrogen systems reported since the year 2000 are compiled in Tab. 2. Together with the measurements carried out within the air-fluorescence community, the results of Pancheshnyi et al. [66] are shown for comparison. Most authors provide values of the characteristic pressures for each $v$ value using the experimental results of several molecular bands with the same upper level $v$. Nagano et al. [47] and the AIRFLY Collaboration [32] report individual results for each measured band. In both cases, $P_{v}^{\prime}$ values reported for bands with the same upper level $v$ are in agreement within the experimental uncertainties, as well as with theoreticval expectations.

While [47] also provides averaged $P_{v}^{\prime}$, AIRFLY recommends using individual experimental results for each wavelength as an empirical result indepen- dent of interpretations at molecular level.

Uncertainties reported by the authors (not quoted in Tab. 2) depend strongly on the intensity of the molecular band. As an example, the uncertainty for the most intense band (337 nm) is below $1 \%$ for the AIRFLY measurement and below $3 \%$ in Nagano et al. [47]. In regard with experiments reporting quenching rate constants for the various upper levels, the uncertainty of AIRLIGHT [50] for $2 \mathrm{P} v=0$ is around 5\% (assuming 4\% uncertainty in the measurement of $K_{Q}$ for nitrogen) and more than $10 \%$ for Pancheshnyi et al. [66].

\subsection{Temperature dependence}

The fluorescence yield $Y_{\lambda}(27)$ depends on temperature through the $P_{v}^{\prime}$ parameter. In general, the $P_{v}^{\prime}$ value for any mixture of gases like air can be written as introduced in (17), (18), and (19). The well known dependence on temperature results from the proportionality between $P_{v}^{\prime}$ and $\sqrt{T}$. Assuming constant density, this results in higher quenching rate constants for increasing temperature because of the molecular Brownian motion.

From molecular physics, a second temperature dependence is expected. The cross sections for collisional quenching $\sigma_{\mathrm{NN}}$ and $\sigma_{\mathrm{NO}}$ depend on the velocity of molecules and therefore their average value vary with temperature. The expected behavior follows a power law in temperature $\sigma \propto T^{\alpha}$, where $\alpha$ is nearly constant in certain temperature intervals (see Sect. 3.2). This $T$ dependence of the collisional cross section had been neglected during the last decades as Bunner claimed an evidence for only a weak temperature dependence [16]. However, as reviewed in [7], considerable effects had been found earlier. Recently, two groups started to measure the collisional cross sections as a function of temperature.

The AIRFLY Collaboration measured at a Van de Graaff electron accelerator with $3.0 \mathrm{MeV}$ kinetic beam energy [44] [68]. The fluorescence signal of dry air has been observed between 284 and $429 \mathrm{~nm}$. A temperature scan with constant density was performed from $240 \mathrm{~K}$ to $310 \mathrm{~K}$ for the $2 \mathrm{P}$ bands $337 \mathrm{~nm}$ (0-0), $354 \mathrm{~nm}(1-2), 314 \mathrm{~nm}(2-1)$, and the $1 \mathrm{~N}$ band $391 \mathrm{~nm}(0-0)$.

In all cases, the fluorescence intensity is found to follow a power law in the given temperature interval. For the $2 \mathrm{P}$ transitions, the fluorescence signal decreases with temperature. According to (22), this behavior implies that $\alpha>-1 / 2$ for the correspond- 
Table 3

Measured temperature dependence parameters for a selected group of air-fluorescence bands as given by AIRFLY [44][68] and Fraga et al. [41]. Additionally, the value extracted from Fig. 6 of [69] for the entire wavelength range is given. A measurement in the $1 \mathrm{~N}$ system had been reported by Lillicrap [70].

\begin{tabular}{|c|c|c|c|c|}
\hline \multirow[b]{3}{*}{$\lambda(\mathrm{nm})$} & \multirow{3}{*}{$\begin{array}{c}\text { AIRFLY [44][68] } \\
\text { in AIR } \\
\alpha_{v}\end{array}$} & Fraga et al. [41] & Grün et al. [69] & Lillicrap $[70$ \\
\hline & & \multicolumn{3}{|c|}{ in NITROGEN } \\
\hline & & $\alpha_{v}$ & $\alpha$ & $\alpha_{v}$ \\
\hline 313.6 & $-0.09 \pm 0.10$ & & & \\
\hline 337.1 & $-0.36 \pm 0.08$ & $-0.87 \pm 0.15$ & & \\
\hline 353.7 & $-0.21 \pm 0.09$ & & & \\
\hline 391.4 & $-0.80 \pm 0.09$ & & & $-0.92^{\mathrm{a}}$ \\
\hline$>300$ & & & $-0.79^{b}$ & \\
\hline
\end{tabular}

a This value is valid in the temperature range between 160 to $300 \mathrm{~K}$. For lower temperature down to $78 \mathrm{~K}, \alpha$ seems to change to -0.41 .

b This value has been obtained from a plot in [69]. The method is very imprecise and we do not know any uncertainties for the data points in the plot. Thus, no uncertainties are provided for the value.

ing upper level. However, the $1 \mathrm{~N}$ fluorescence signal increases with $T$ and, thus, $\alpha<-1 / 2$. The result of a fit to the experimental data is shown in Tab. 3.

Another experiment on the temperature dependence was performed by Fraga et al., using pure nitrogen [41]. They use $\alpha$-particles from a ${ }^{241} \mathrm{Am}$ radioactive source. After several thorough tests of systematics, the temperature dependence of the $337 \mathrm{~nm}$ band of nitrogen was analyzed. During a temperature scan, the density was kept constant as in the experiments from AIRFLY [44]. A fit to the measured data points yields a value of $\alpha=-0.87 \pm 0.15$ consistent with an increasing quenching cross section with decreasing temperature. This result is also reviewed in Tab. 3 for comparison.

The table also reports the measurements of older experiments in pure nitrogen carried out by Grün and Schopper [69] as well as Lillicrap [70]. Grün and Schopper measured the $T$ dependence for the integral spectrum ( $>300 \mathrm{~nm}$ ). The $\alpha$-value reported in Tab. 3 has been obtained from Fig. 6 of [69]. The result of $\alpha=-0.79$ is consistent with that of Fraga et al., that is, fluorescence increases with $T$ at a similar rate. On the other hand, Lillicrap reported measurements for the $1 \mathrm{~N}(0-0)$ band $(391 \mathrm{~nm})$. In the range $160-300 \mathrm{~K}$ the temperature dependence indicates an $\alpha$-value of about -0.92 , while at lower temperature $(78-160 \mathrm{~K}) \alpha$ seems to increase up to about -0.41 . The AIRFLY measurements for the $1 \mathrm{~N}(0-0)$ band on air cannot be compared with that of Lillicrap for pure nitrogen. As pointed out in Sect. 3.2, the $\alpha$-parameter depends on the nature of the partners and the type of interaction. In principle, its value for $\mathrm{N}-\mathrm{O}$ collisions is expected to be different than that for N-N collisions. Since oxygen is a much more efficient quencher, the effect of $\mathrm{N}-\mathrm{O}$ collisions is expected to dominate in the temperature dependence of the air-fluorescence light. Similarly, a comparison between the results for the $337 \mathrm{~nm}$ band $2 \mathrm{P}(0-0)$ of Fraga et al. and AIRFLY is not possible.

In [71], the resulting fluorescence emission in units of photons /m of a $0.85 \mathrm{MeV}$ electron in the Earth's atmosphere is shown for the AIRFLY data and Grün and Schopper data. Because of an additional 1/Tdependence in the density-multiplication to calculate the fluorescence emission in photons $/ \mathrm{m}$, the effect of temperature-dependent collisional cross sections is strongest for the Grün and Schopper data. However, a caveat has to be applied to this comparison: the $\alpha$-parameter from Grün and Schopper has been measured in pure nitrogen and is used there also for nitrogen-oxygen quenching.

In summary, recent measurements confirm a temperature dependence of collisional cross sections. Quenching cross sections decrease with increasing temperature for both nitrogen-nitrogen and nitrogen-oxygen collisions and for both molecular systems, $1 \mathrm{~N}$ and 2P. Only the AIRFLY Collaboration provides results for air. The comparison with measurements on pure nitrogen is difficult since no information is available on cross sections for $\mathrm{N}-\mathrm{O}$ collisions. Further investigations are recommended because a relevance for cosmic-ray measurements is indicated. Especially, measurements performed in one set-up with both gases, nitrogen and air, as well as for representative bands of the $2 \mathrm{P}$ and $1 \mathrm{~N}$ 
system of nitrogen are highly welcome to study the temperature-dependent collisional cross section for nitrogen-nitrogen and nitrogen-oxygen quenching.

\subsection{Humidity dependence}

Water vapor is an always changing constituent of the Earth's atmosphere. With respect to the fluorescence emission, the $\mathrm{H}_{2} \mathrm{O}$ molecules serve as an additional quenching partner for the excited $\mathrm{N}_{2}$ molecules. Hence, (17) has to be extended by a term accounting for collisional quenching due to water vapor.

Measurements on water vapor quenching have been performed and reported at the 5th Fluorescence Workshop by AIRFLY [44][68], Sakaki et al. [72], and Waldenmaier et al. (AIRLIGHT) [50] [73]. Somewhat earlier, measurements have been reported by Morozov et al. [56], Pancheshnyi et al. [67][66], see e.g. [7], and the MACFLY Collaboration $[52]$.

The AIRFLY group measured the humidity dependence at a Van de Graaff electron accelerator [44][68], as they did it for the temperature, see Sect. 5.2. The fluorescence chamber was filled with high purity dry air at atmospheric pressure. Before the gas entered the chamber, it was flown through a bubbler containing high purity water. The relative humidity in the chamber was regulated from 0 to $100 \%$ which is about $25 \mathrm{hPa}$ partial pressure under their experimental conditions. In order to describe the water vapor quenching, AIRFLY used the modified function for $1 / P_{\text {hum }}^{\prime}$, see $(20)$. They reported values for $P_{\mathrm{H}_{2} \mathrm{O}}^{\prime}$ for the wavelengths $314 \mathrm{~nm}, 337 \mathrm{~nm}$, $354 \mathrm{~nm}$, and $391 \mathrm{~nm}$, see Tab. 4. This additional source of quenching has a non-negligible effect, since the fluorescence yield decreases by about $20 \%$ for a relative humidity of $100 \%$ at atmospheric pressure and room temperature.

Sakaki et al. excited humid air with electrons from a ${ }^{90} \mathrm{Sr}$ source with $3.7 \mathrm{MBq}$, so that each run lasted about 1 week [72]. The fluorescence yield was measured as function of specific humidity for two bands of the $2 \mathrm{P}$ system $(337 \mathrm{~nm}, 358 \mathrm{~nm})$ and the most intense $1 \mathrm{~N}$ band $(391 \mathrm{~nm})$. A function $\varepsilon(P)=C \times$ $P /\left(1+P / P^{\prime}\right)$ is fitted to the data with $C$ and $P^{\prime}$ as fitting parameters. This procedure allowed the authors to find laws of photon yield versus altitude directly applicable to air shower analyses.

Another experiment with ${ }^{90} \mathrm{Sr}$ was performed by Waldenmaier et al. [50]. Here the activity of the source was $37 \mathrm{MBq}$ resulting in runs of about 30 hours. Only the last 20 hours were used in the analysis to ensure stable conditions of the humidity in the chamber. Overall, six runs at $15-17^{\circ} \mathrm{C}$ and $30 \mathrm{hPa}$ of pure nitrogen were obtained. Waldenmaier et al. provide water vapor quenching rate constants for $2 \mathrm{P}(v=0,1)$ and $1 \mathrm{~N}(v=0)$, see Tab. 4 .

A comparison of the obtained values with Morozov et al. [56] and Pancheshnyi et al. [67][66] shows that the quenching rate constant from AIRLIGHT lies in between these two. The values from AIRFLY agree quite well with those from Morozov et al. The relatively large values of Pancheshnyi et al. might be due to their method of mixing nitrogen with water vapor [56]. In their set-up oxygen and hydrogen were added to the nitrogen gas and for the analysis it was assumed that the admixtures to nitrogen were completely converted to water vapor in the cell. If not all hydrogen and oxygen had been converted to water vapor, the actual water vapor pressure could have been lower than expected, resulting in too high $P_{\mathrm{H}_{2} \mathrm{O}}^{\prime}$ values. Also the $P_{\mathrm{H}_{2} \mathrm{O}}^{\prime}$ values from MACFLY [52] are about twice the values from AIRFLY resulting in less quenching and hence higher fluorescence yield compared to AIRFLY. In the MACFLY experiment the quenching rate constants have been calculated using a combination of experimental data and molecular constants from results published previously.

\subsection{Argon effect}

Argon contributes to the Earth's atmosphere with $0.93 \%$ per volume. With respect to the nitrogen fluorescence emission, three possible effects of argon have to be considered: energy transfer from argon to nitrogen, direct fluorescence light emitted by argon, and collisional quenching of excited nitrogen molecules with argon.

Argon can be excited from electrons by

$$
\mathrm{e}+\mathrm{Ar} \rightarrow \mathrm{Ar}^{*}
$$

where the excitation cross section is largest for $\operatorname{Ar}\left({ }^{3} P_{2}\right)$ [74]. This excitation of argon is followed by an efficient energy transfer from argon to nitrogen [69] via

$$
\mathrm{Ar}^{*}+\mathrm{N}_{2} \rightarrow \mathrm{Ar}+\mathrm{N}_{2}^{*}\left(C^{3} \Pi_{u}\right) .
$$

The reached excited state of nitrogen is the known upper level of the second positive system.

Under certain conditions, mixtures of argon with water vapor might emit ultraviolet radiation at 
Table 4

Values for $P_{\mathrm{H}_{2} \mathrm{O}}^{\prime}$ as measured by several experiments. Since different experiments used different techniques to obtain these values and some of them quote statistical uncertainties only, here no uncertainties are reviewed. Directly, the values are incommensurable, thus, we ask the reader to refer to the uncertainties in the original publications. All measurements were performed at room temperature varying from about $288 \mathrm{~K}$ up to $300 \mathrm{~K}$.

\begin{tabular}{lccccc}
\hline & AIRFLY [44] & AIRLight [50] ${ }^{\mathrm{a}}$ & $\begin{array}{c}\text { Morozov et al. [56 }{ }^{\mathrm{a}} \\
{ }^{2}\end{array}$ & $P_{\mathrm{H}_{2} \mathrm{O}}(\mathrm{hPa})$ \\
\hline $2 \mathrm{P}(v=0)$ & 1.28 & 1.92 & 1.31 & 2.47 & 2.94 \\
$2 \mathrm{P}(v=1)$ & 1.27 & 2.13 & 1.39 & 2.67 & 2.63 \\
$2 \mathrm{P}(v=2)$ & 1.21 & & & 2.59 & 2.55 \\
$2 \mathrm{P}(v=3)$ & & & & 2.19 & 2.25 \\
$1 \mathrm{~N}(v=0)$ & 0.33 & 0.39 & 0.76 & 0.76 \\
\hline
\end{tabular}

${ }^{\text {a }}$ Inferred from measurements of quenching rate constants and lifetimes .

around $310 \mathrm{~nm}$. The main contribution comes from the transition $\mathrm{OH} A^{2} \Sigma^{+} \rightarrow X^{2} \Pi \quad[75]$. A detailed study of this transition has shown highest intensities for very low argon pressure and $0.06 \mathrm{~Pa}$ water vapor. Because of these special conditions, the fluorescence emission of this transmission will be of no importance for the observation of extensive air showers.

The increase of emission by energy transfer from argon to nitrogen competes, however, with a higher quenching rate. The non-radiative de-excitation of nitrogen is caused by additional collisions of nitrogen with argon atoms in air.

Already very early Bunner stated the net effect of argon to be less than $1 \%$ contribution to the fluorescence light [16]. More recently, AIRFLY measured the effect of argon while comparing the fluorescence yield from nitrogen-oxygen mixture with dry air. They found that the effect of argon is completely negligible at atmospheric pressure [29].

\subsection{Energy dependence}

Theoretical results on the proportionality between fluorescence intensity and deposited energy have been presented above (Sect.4.2.2). These predictions [55] indicate that the fluorescence yield is basically independent of the electron energy for the typical experimental situations in this field. Nevertheless, experimental tests of the proportionality between deposited energy and fluorescence intensity are mandatory.

Several groups provided data on this topic. Two different experimental methods have been used for this purpose. Firstly, in a thick-target configuration the fluorescence intensity measured as function of the shower depth is compared with the energy deposited by the shower, either directly measured with an appropriate device or calculated with a Monte Carlo code (e.g. EGS4, GEANT). Secondly, measurements of the fluorescence yield in a thin-target experiment for various electron energies provide the required data to test this proportionality. Notice that in the latter technique it is necessary to measure the fluorescence yield for well separated electron energies.

The FLASH collaboration measured the air fluorescence yield as a function of the shower depth in a thick-target experiment for the first time [59]. As mentioned above, this experiment used $28.5 \mathrm{GeV}$ electrons to induce an electromagnetic shower with a composition similar to that generated by a $10^{18} \mathrm{eV}$ cosmic ray. The authors found that the ratio of measured photomultiplier signals to deposited energy is constant in the full shower depth range $(2-14$ radiation lengths) within 5\% uncertainty (Fig. 12). The dependence of deposited energy on the shower development stage, measured by an ion chamber, was found in good agreement with a simulation carried out with EGS4. Fig. 7 of [58] shows the relative value of the fluorescence intensity as a function of depth for several band pass filters. The fluorescence intensity fits well the predictions of an empirical deposition model, see (1) in [58].

Several experiments have carried out direct measurements of the fluorescence yield as a function of energy. The MACFLY experiment measured the fluorescence yield at $1.5 \mathrm{MeV}, 20 \mathrm{GeV}$, and $50 \mathrm{GeV}$ using the same collision chamber. MACFLY reports values of 17.0, 17.4, and 18.2 photons/MeV, respectively, with uncertainties of about $13 \%$. In summary, the MACFLY thin-target experiment found that the 


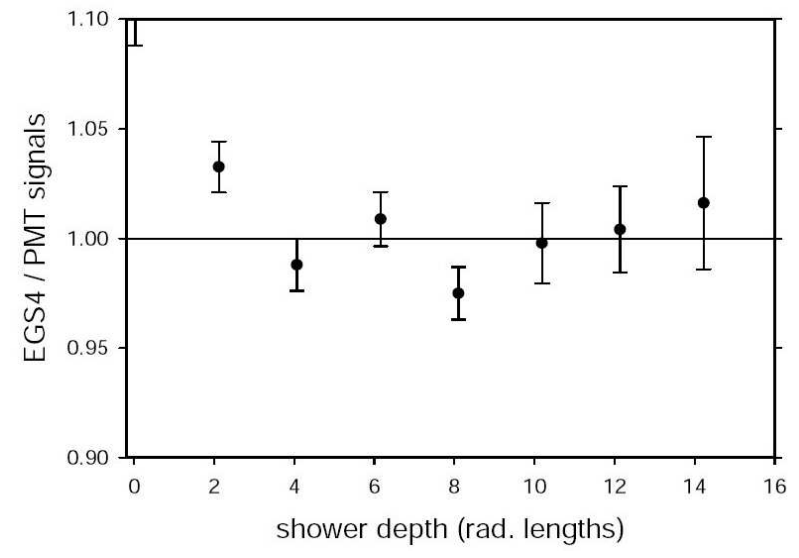

Fig. 12. Ratio of EGS4 predictions to weighted average of photomultiplier signals versus shower depth in the thick-target experiment of the FLASH Collaboration [58].

fluorescence yield is independent of energy in the interval $1.5 \mathrm{MeV}-50 \mathrm{GeV}$. Although no measurement was carried out for other energies inside this large interval, no theoretical prediction suggests a lack of proportionality inside this wide interval.

The MACFLY Collaboration carried out a thicktarget experiment [60] which supports the above result. The MACFLY thick-target experiment finds the fluorescence yield to be proportional to the deposited energy for all stages of showers initiated by high-energy electrons following a technique similar to that of FLASH.

The AIRFLY Collaboration makes use of various accelerators to measure the fluorescence yield in several energy intervals (see Sect.4.1.1 for more details). The obtained values for the fluorescence yield are compared with the deposited energies calculated with a full GEANT4 simulation of the experiment [53]. Since the inter-calibration between different energy intervals has not yet been achieved, the result is only valid inside energy intervals.

In the first place, the interval $0.5-15 \mathrm{MeV}$ is covered in two subintervals $0.5-3 \mathrm{MeV}$ and $3-15 \mathrm{MeV}$. In both cases the fluorescence signal is proportional to deposited energy. In the second one, the relativistic increase is clearly observed in the fluorescence signal. Since $3 \mathrm{MeV}$ energy is included in both subintervals, the proportionality test covers the whole interval $0.5-15 \mathrm{MeV}$. A deviation from perfect proportionality lower than $3 \%$ is reported by AIRFLY in this interval. For the interval $50-420 \mathrm{MeV}$, measurements of the fluorescence yield turn out to be proportional to the deposited energy also within 3\% uncertainty. Finally, the range $6-30 \mathrm{keV}$ is studied, finding proportionality within $5 \%$.

In summary, AIRFLY shows that the fluorescence yield is independent of electron energy within several energy intervals. Since these intervals are very large, the AIRFLY data provide a good experimental test of the proportionality assumption which should be completed in the future by means of an absolute calibration in the whole energy range.

Other experiments reported proportionality between deposited energy and fluorescence intensity although within much smaller energy intervals. The AIRLIGHT experiment [50] did not find any noticeable dependence of the fluorescence yield on electron energy in the range $0.25-2.00 \mathrm{MeV}$. This test was carried out for several molecular transitions in a pressure range of $50-800 \mathrm{hPa}$. As will be mentioned below, these authors carried out a detailed simulation using GEANT4 to determine the energy deposited in the chamber.

Kakimoto et al. [45] compared the fluorescence intensity measured in photons $/ \mathrm{m}$ with the electron energy loss in air for several energies in the range 1.4 MeV - 1.0 GeV finding proportionality. A similar comparison of the photon yield (photons $/ \mathrm{m}$ ) of Nagano et al. with the above results of Kakimoto et al. leads to the same conclusion [46]. However, notice that in this case the assumption has to be made that all energy lost by the electrons is deposited within the field of view of the optical system.

\subsection{Absolute yield}

The available results on absolute values of the airfluorescence yield are summarized in Tab. 5. As mentioned in Sect. 3.3, different parameters can be used to account for the fluorescence intensity emitted by an electron beam in air. Some experiments report results on the number of fluorescence photons per electron and unit path length, i.e. the $\varepsilon_{\lambda}\left[\mathrm{m}^{-1}\right]$ value, while other publications give the result in number of photons per unit of deposited energy, i.e. the definition of fluorescence yield $Y_{\lambda}$ in this article. Different experiments measure the fluorescence intensity in different spectral intervals ranging from narrow band (e.g. $337 \mathrm{~nm}$ ) to wide spectral intervals (e.g. $290-440 \mathrm{~nm}$ ). In addition, very often results are referred to different pressures and/or temperatures.

Kakimoto et al. [45] reported a value for the fluorescence efficiency for the $337 \mathrm{~nm}$ band of $\Phi_{337}=$ $2.1 \times 10^{-5}$ at $800 \mathrm{hPa}$ and $288 \mathrm{~K}$. Therefore, the fluorescence yield at these conditions equals $5.7 \mathrm{MeV}^{-1}$. 
Table 5

Experimental results on absolute values of the air-fluorescence yield at room temperature and atmospheric pressure in the wavelength interval shown in the third column. Measurements are carried out at the energy given in the second column. Results are split depending on the units used by the authors. For more details see text.

\begin{tabular}{|c|c|c|c|c|c|c|c|c|}
\hline \multirow[t]{3}{*}{ Experiment } & \multirow[b]{3}{*}[\mathrm{MeV}]{} & \multirow{3}{*}{$\begin{array}{c}\lambda \\
{[\mathrm{nm}]}\end{array}$} & \multirow{3}{*}{$\begin{array}{c}\mathrm{P} \\
{[\mathrm{hPa}]}\end{array}$} & \multirow{3}{*}{$\begin{array}{c}\mathrm{T} \\
{[\mathrm{K}]}\end{array}$} & \multicolumn{4}{|c|}{ Fluorescence yield } \\
\hline & & & & & \multicolumn{2}{|c|}{ wide spectrum } & \multicolumn{2}{|c|}{$337 \mathrm{~nm}$} \\
\hline & & & & & {$\left[\mathrm{m}^{-1}\right]$} & {$\left[\mathrm{MeV}^{-1}\right]$} & {$\left[\mathrm{m}^{-1}\right]$} & {$\left[\mathrm{MeV}^{-1}\right]$} \\
\hline AIRFLY [62] & 350 & 337 & 993 & 291 & & & & $4.12^{\mathrm{a}}$ \\
\hline FLASH [51] & $2.85 \times 10^{4}$ & $300-420$ & 1013 & 304 & & 20.8 & & \\
\hline Lefeuvre et al. [54] & 0.85 & $300-430$ & 1013 & 288 & 4.23 & & & \\
\hline \multirow[t]{2}{*}{ Nagano et al. [47] } & 0.85 & $300-406$ & 1013 & 293 & 3.81 & & 1.02 & 5.03 \\
\hline & & $300-430$ & & & 4.05 & & & \\
\hline \multirow[t]{3}{*}{ MACFLY [52] } & 1.5 & $290-440$ & 1013 & 296 & 3.14 & 17.6 & & \\
\hline & $2.0 \times 10^{4}$ & & & & 4.22 & & & \\
\hline & $5.0 \times 10^{4}$ & & & & 4.44 & & & \\
\hline AIRLIGHT [50] & $0.25-2.00$ & 337 & 1013 & 293 & & & & 5.68 \\
\hline Kakimoto et al. [45] & $1.4-1000$ & $300-400$ & 800 & 288 & & & & 5.7 \\
\hline
\end{tabular}

a Preliminary, since the final absolute calibration is pending.

Kakimoto et al. used for the computation of $\Phi_{337}$ the energy loss of the electron in the chamber as a measure of the deposited energy.

Nagano et al. [47] provide the absolute number of photons per meter at $293 \mathrm{~K}$ and an average energy of $0.85 \mathrm{MeV}$ for individual molecular bands, in particular the $337 \mathrm{~nm}$ one as well as the integral value in two spectral intervals of interest $(300-406 \mathrm{~nm}$ and 300 $-430 \mathrm{~nm}$ ). A fluorescence yield for the $337 \mathrm{~nm}$ band of 5.03 photons/MeV at $293 \mathrm{~K}$ and $1013 \mathrm{hPa}$ is easily inferred from the $\Phi_{337}^{0}$ and $P_{337}^{\prime}$ values reported in this work. In principle, the possible effect of secondaries escaping the field of view (see Sect.4.1.4) is not treated in this work. The quoted uncertainty is $13 \%$.

At the same average energy Lefeuvre et al. [54] report a value of 4.23 photons $/ \mathrm{m}$ in the range 300 - $430 \mathrm{~nm}$ at $288 \mathrm{~K}$ and $1013 \mathrm{hPa}$. These measurements lack from a systematic study of the pressure dependence of the fluorescence yield and, therefore, they cannot provide $P^{\prime}$ values. Although the wavelength spectrum was registered using a monochromator, relative intensities were not measured and, consequently, the absolute value of the fluorescence yield for the $337 \mathrm{~nm}$ band was not reported. According to their calculations, a very small correction for the effect of lost secondary electrons has to be applied. The authors claim to have achieved an extremely high accuracy (5.0\% uncertainty).

The AIRLIGHT experiment [50] provides results on the quenching rate constants and lifetimes for several molecular bands. The energy deposited in the chamber was calculated using GEANT4 showing that a non-negligible correction is necessary, in particular, at high energy and high pressure. The authors give results on the fluorescence yield at zero pressure with a systematic uncertainty of about $15 \%$ which, combined with quenching rate constants and lifetimes, allows to determine the absolute value of the air-fluorescence yield for any pressure and temperature for several molecular bands. As an example, the predicted value at $293 \mathrm{~K}$ and atmospheric pressure for the $337 \mathrm{~nm}$ band is shown in Tab. 5 .

Three experiments have carried out absolute measurements of the air-fluorescence yield using highenergy electrons. The FLASH Collaboration, working with $28.5 \mathrm{GeV}$ electrons, reports absolute values in the spectral range $300-420 \mathrm{~nm}$ at several pressures in the interval $67-1013 \mathrm{hPa}$ and $304 \mathrm{~K}$ temperature. The value at atmospheric pressure is 20.8 photons/MeV. The authors performed an absolute calibration by comparison with the Rayleigh scattering from a nitrogen laser. An uncertainty of this absolute value below $8 \%$ is reported.

The MACFLY Collaboration measures the absolute fluorescence yield in the spectral range 290 $440 \mathrm{~nm}$ at $296 \mathrm{~K}$. The authors report results in photons $/ \mathrm{m}$ at three electron energies, $1.5 \mathrm{MeV}, 20 \mathrm{GeV}$, and $50 \mathrm{GeV}$. A comparison of these numbers with the energy deposited in the chamber according to a 
GEANT4 simulation leads to an averaged fluorescence yield value of 17.6 photons $/ \mathrm{MeV}$ for the above mentioned spectral range and temperature. According to the authors, the uncertainty of this result is about $15 \%$.

Finally, the AIRFLY Collaboration carried out systematic studies on the dependence of the fluorescence yield on pressure and electron energy. As mentioned above, this collaboration has developed a novel method for the absolute calibration of the experimental system based on the comparison with Cherenkov radiation generated by the electron beam. The AIRFLY Collaboration carried out a detailed GEANT4 simulation to determine accurately the energy deposited inside the field of view of the optical system. A careful analysis of the various contributions to the systematic uncertainties leads them to conclude conservatively that reducing the uncertainty below the $10 \%$ level is achievable. Using an electron beam of $350 \mathrm{MeV}$, a preliminary result of 4.12 photons $/ \mathrm{MeV}$ for the $337 \mathrm{~nm}$ band at $291 \mathrm{~K}$ is given. Further measurements and checks will lead in a near future to a final result for the absolute value.

A comparison of experimental results when expressed in different units is not straightforward. Assuming a fixed wavelength interval, pressure and temperature, results in photons $/ \mathrm{m}$ could be translated to photons/MeV as far as the deposited energy (within the field of view of the optical system) per unit path length and electron is known.

Results on this parameter have been calculated using EGS4 or GEANT4 for several experimental configurations [52][51]. In [55], a simulation at microscopic level gives results in good agreement with those mentioned above. The results of [55] indicate that deposited energy per unit column density is only smoothly dependent on the product of pressure and radial size of the observation volume.

For the comparison of different measurements in the same units, assuming similar geometry, but in different spectral ranges, the relative intensities and the characteristic pressures are needed.

In [55], a procedure for the comparison of absolute values expressed in different units and for different spectral ranges based on the above arguments is shown. Since data on relative intensities, $P_{v}^{\prime}$ values, and deposited energy per meter in a particular configuration have uncertainties, this procedure does not allow to compare high-accuracy measurements, nevertheless it can be useful for a comparison of experimental data at the level of about $15 \%$ uncertainty.

\section{The fluorescence light yield in the atmosphere}

For cosmic-ray experiments, the measurement of the nitrogen fluorescence emission is the most direct method to detect the longitudinal profile of extensive air showers. For the event reconstruction procedures of these air shower experiments, the knowledge of the fluorescence yield and its dependence on atmospheric conditions are crucial parameters. The principle of air shower detection with fluorescence light has been discussed above (Sect. 2.1). The most relevant altitude range for ultra high-energy cosmic rays is between ground level and about $13 \mathrm{~km}$ above sea level (a.s.l.). The shower maximum is reached between 2 and $8 \mathrm{~km}$ a.s.l. for a shower with $10^{19} \mathrm{eV}$, depending on type and inclination angle of the primary particle. The field of view of the fluorescence telescopes of air shower experiments covers this range. For example, the Auger telescopes oversee the sky between $0.7 \mathrm{~km}$ and $12.5 \mathrm{~km}$ above the altitude of the Pierre Auger Observatory, 1.4 km a.s.l., at a distance of $20 \mathrm{~km}$.

Up to at least $80 \mathrm{~km}$ a.s.l., it is safe to assume a constant composition of the Earth's atmosphere which is mainly $78.08 \% \mathrm{~N}_{2}, 20.95 \% \mathrm{O}_{2}$, and $0.93 \%$ Ar per volume. All three constituent parts influence the emission of fluorescence light, however, with strongly differing importance. As already discussed throughout this summary article, emission from nitrogen is the dominant light. The contribution of argon has been discussed in Sect. 5.4. The UVfluorescence light emission from $\mathrm{O}_{2}$ is negligible [76]. The contribution between 300 and $400 \mathrm{~nm}$ stems from $\mathrm{O}_{2}^{+} A^{2} \Pi_{u}-X^{2} \Pi_{g}$ transitions. However, their intensities are negligible as compared with those of nitrogen. The emission of atomic oxygen has wavelengths larger than $395 \mathrm{~nm}$, up to $845 \mathrm{~nm}$ [76], but with no relevance for air shower experiments.

To compare the fluorescence light profiles in the Earth's atmosphere, in former discussion often an electron energy of $0.85 \mathrm{MeV}$ has been chosen. These electrons lose their energy mainly by ionization and the energy deposit is $d E / d X=$ $0.1677 \mathrm{MeV} / \mathrm{kg} \mathrm{m}^{-2}$ [46]. To obtain a light profile induced by an extensive air shower, the longitudinal profile of the locally deposited energy $E_{\text {dep }}$ per $\mathrm{g} \mathrm{cm}^{-2}$ has to be known. A resultant number of fluorescence photons $\varepsilon_{\lambda}$ with wavelength $\lambda$ per unit 


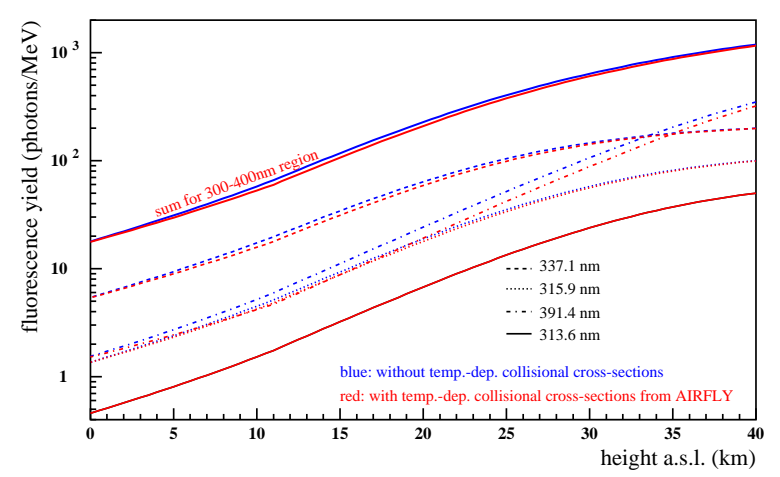

Fig. 13. Fluorescence yield profile for different wavelengths in the US Standard Atmosphere. The blue curves represent the fluorescence yield accounting for the $\sqrt{T}$-dependence only, compare to Fig. 3 in [78]. The red curves account for the entire temperature dependence, thus including the temperature-dependent collisional cross sections [71], with parametrization given from AIRFLY [68].

path length is then given as

$$
\varepsilon_{\lambda}=Y_{\lambda} \cdot \frac{\lambda}{h c} \cdot E_{d e p} \cdot \rho_{a i r}
$$

The air density $\rho_{\text {air }}$ is that at the position of the energy deposit, thus, where the photons are emitted. $Y_{\lambda}$ is the fluorescence yield including all temperature, pressure, and humidity dependences as discussed in Sects. 5.2, 5.5, and 5.3, respectively. With regard to extensive air showers, the transition from the fluorescence yield $Y_{\lambda}$ in photons emitted per deposited energy to the number of fluorescence photons $\varepsilon_{\lambda}$ per unit path length is straightforward because all secondary electrons involved in the fluorescence process are in the observed volume. No boundary effects as for laboratory measurements have to be considered as discussed in Sect. 4.2.

Before this workshop, only the $\sqrt{T}$-dependence and the pressure dependence have been taken into account. In the Earth's atmosphere as described in the US Standard Atmosphere [77], the temperature decreases up to $11 \mathrm{~km}$ a.s.l. with a continuous lapse rate of $6.5 \mathrm{~K} / \mathrm{km}$. For higher altitudes, until $20 \mathrm{~km}$ a.s.l., the temperature remains constant. Above, the temperature increases again until the stratopause at around $50 \mathrm{~km}$ a.s.l. This temperature profile, together with the pressure profile of the atmosphere, affects the fluorescence yield in the atmosphere differently for each band system due to different deactivation constants. In Fig. 13, the blue curves represent the fluorescence yield accounting for the $\sqrt{T}$-dependence [78].

A detailed calculation of the fluorescence emis-

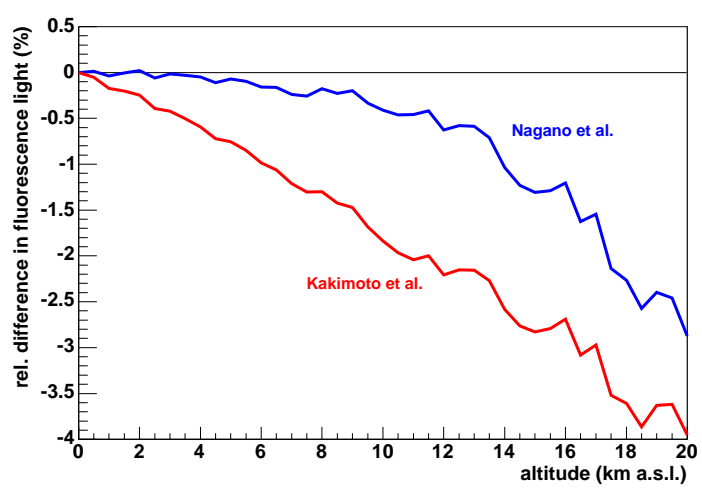

Fig. 14. Relative difference for the fluorescence light profiles in the US Standard Atmosphere with a $0.85 \mathrm{MeV}$ electron as incident particle of the two parametrizations, (34) and (29) compared with the detailed calculation of [78].

Table 6

$A$ and $B$ values in 10 bands between 300 and $400 \mathrm{~nm}$ as given by Nagano et al. [47].

\begin{tabular}{ccc}
\hline Main $\lambda(\mathrm{nm})$ & $A\left(\mathrm{~m}^{2} \mathrm{~kg}^{-1}\right)$ & $B\left(\mathrm{~m}^{3} \mathrm{~kg}^{-1} \mathrm{~K}^{-1 / 2}\right)$ \\
\hline 316 & $20.5 \pm 1.3$ & $2.14 \pm 0.18$ \\
329 & $3.91 \pm 0.35$ & $1.22 \pm 0.14$ \\
337 & $45.6 \pm 1.2$ & $2.56 \pm 0.10$ \\
354 & $3.68 \pm 0.39$ & $1.60 \pm 0.21$ \\
358 & $37.8 \pm 2.3$ & $2.72 \pm 0.22$ \\
376 & $6.07 \pm 0.57$ & $1.44 \pm 0.17$ \\
381 & $12.7 \pm 1.4$ & $2.53 \pm 0.35$ \\
391 & $50.8 \pm 2.1$ & $9.80 \pm 0.51$ \\
394 & $2.25 \pm 0.78$ & $2.03 \pm 0.79$ \\
400 & $4.58 \pm 0.44$ & $2.03 \pm 0.23$ \\
\hline
\end{tabular}

sion including atmospheric effects with constant collisional cross sections $\sigma_{\mathrm{N} x, v}$ has been compared in Keilhauer et al. [78] with parametrizations of the altitude dependence. Nagano et al. provided parameters $A_{\lambda}$ and $B_{\lambda}$ for all 10 wavelengths they measured between 300 and $400 \mathrm{~nm}$, see Tab. 6, describing the fluorescence light emission per unit path length as $[47]$

$$
\varepsilon_{\lambda}=E_{d e p} \cdot\left(\frac{A_{\lambda} \rho}{1+\rho B_{\lambda} \sqrt{T}}\right)
$$

Kakimoto et al. reported only one set of parameters $A_{1,2}$ and $B_{1,2}$, see Tab. 12 in [7], in order to describe the entire wavelength range with (29). Comparing only the altitude dependence in the US Standard Atmosphere [77], assuming an equal absolute number of fluorescence photons at ground, shows a quite good agreement, see Fig. 14. 


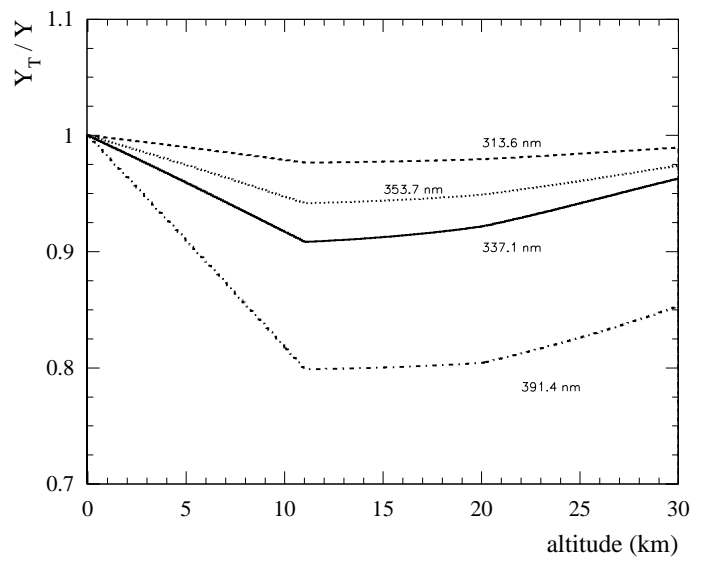

Fig. 15. Ratio of fluorescence yield with measured $\alpha_{\lambda}$ to the one with $\alpha_{\lambda}=0$ : dashed line $313.6 \mathrm{~nm}$; full line $337.1 \mathrm{~nm}$; dotted line $353.7 \mathrm{~nm}$; dashed-dotted line $391.4 \mathrm{~nm}$ [44].

A further analysis of the altitude dependence has been performed for the atmospheric conditions at the site of the Pierre Auger Observatory [78]. Four seasonal atmospheric models have been developed. The fluorescence light induced by a $0.85 \mathrm{MeV}$ electron, applying the well-known dependences, has been compared to that expected in the US Standard Atmosphere, see Fig. 5 in [71] ${ }^{10}$. The differences for the Argentine seasons compared with the US Standard Atmosphere are well below $\pm 5 \%$ [78].

Introducing also the effect of temperaturedependent collisional cross sections, see Sect. 5.2, the reduction of the fluorescence yield is significant. The red curves in Fig. 13 are the fluorescence yield accounting for both temperature effects with data from AIRFLY. The relative differences of the yield with both effects compared to the fluorescence yield just with the $\sqrt{T}$-dependence can be seen in Fig. 15 . The overall reduction for the wavelength range between 300 and $400 \mathrm{~nm}$ is dominated by the reduction of the $337 \mathrm{~nm}$ band up to $18 \mathrm{~km}$ a.s.l., so that these two curves would lie on top of each other. For higher altitudes, the contributions of other bands become more important and the overall reduction increases compared to that of the $337 \mathrm{~nm}$ band.

Applying this dependence to the calculation of the light emission profiles of extensive air showers, the expected shower light profile of an Fe-induced cascade with $10^{19} \mathrm{eV}$ is reduced by $2.7 \%$ up to $7.5 \%$ depending on inclination of the shower and the at-

\footnotetext{
${ }^{10}$ In the nomenclature of this summary article, the fluorescence yield shown there corresponds to $\varepsilon_{\lambda}$.
}

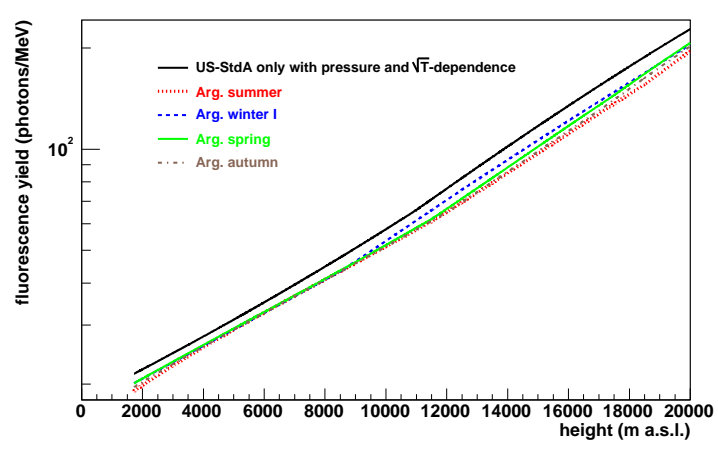

Fig. 16. Fluorescence yield $Y_{\lambda}$ in the US Standard Atmosphere only with pressure and $\sqrt{T}$-dependence together with the fluorescence yield as expected in Argentine atmospheres accounting for all newly known dependences. The temperature-dependent collisional cross sections are taken from AIRFLY [44][68] and the collisional cross sections due to water vapor are measured by Waldenmaier et al. [50][73].

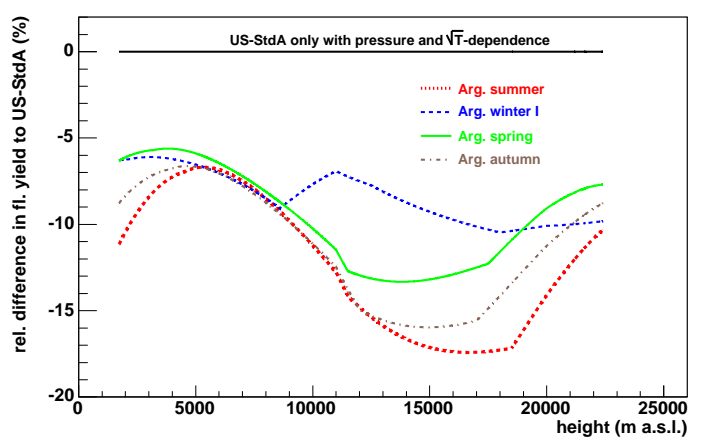

Fig. 17. Relative difference of the fluorescence yield $Y_{\lambda}$ in Argentine atmospheres with the latest results of pressure, temperature, and humidity dependences to that in the US Standard Atmosphere only with pressure and $\sqrt{T}$-dependence.

mospheric model [71]. The values given here refer to Argentine seasonal atmospheric models as used for the Pierre Auger Observatory [13].

These seasonal atmospheric models have been used to study further the altitude dependence of the fluorescence yield. In order to include the humidity dependence, seasonal average profiles of the relative humidity at the site of the Pierre Auger Observatory have been obtained by meteorological radio soundings during night-time [71]. As a result of the newly measured temperature and humidity dependences, Sect. 5.2 and 5.3, combined with the well-known dependences on temperature and pressure, also Sect. 5.2 and 5.5, the fluorescence yield $Y_{\lambda}$ changes significantly in the Earth's atmosphere. Fig. 16 displays $Y_{\lambda}$ in the US Standard Atmosphere 


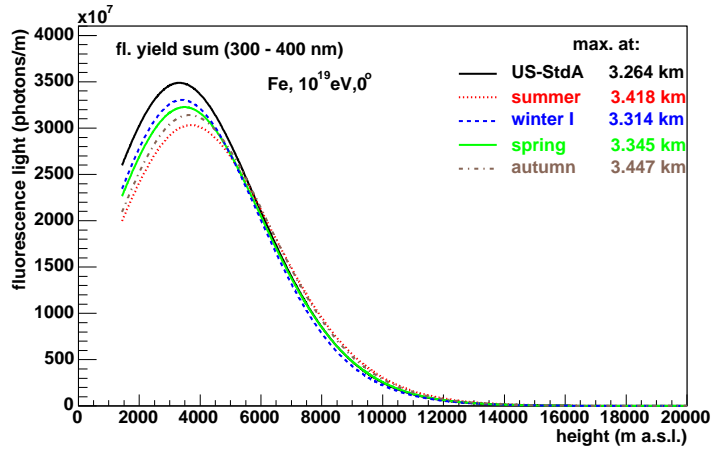

Fig. 18. Fluorescence light profiles for average iron-induced air showers with $10^{19} \mathrm{eV}$ with vertical incidence [71]. For the profiles in Argentine atmospheres, all newly measured altitude dependences are taken into account. For the US Standard Atmosphere only the pressure and $\sqrt{T}$-dependence have been considered.

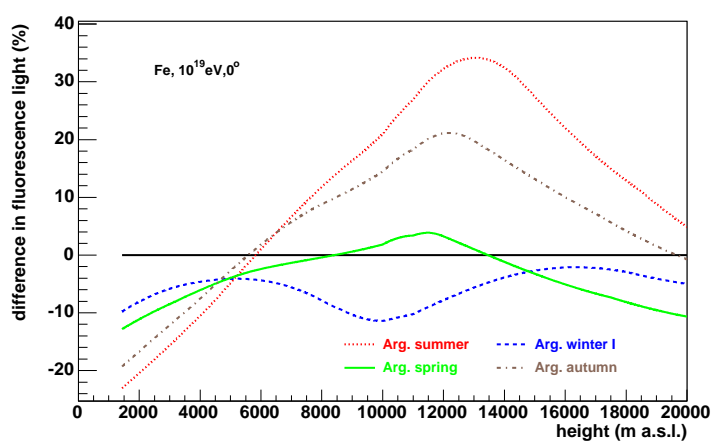

Fig. 19. Difference of the fluorescence light profiles in Argentine atmospheres to that in the US Standard Atmosphere as shown in Fig. 18 [71].

only with pressure and $\sqrt{T}$-dependence together with the fluorescence yield as expected in Argentine atmospheres accounting for all newly known dependences. The relative difference of the fluorescence yield with the latest results to that in the US Standard Atmosphere with the well-known dependences is of the order of 10\%, as can be seen in Fig. 17.

To estimate the importance of the newly measured altitude dependences on reconstructing shower profiles, average iron-induced air showers with $E_{0}=10^{19} \mathrm{eV}$ have been simulated with CORSIKA [79] in [71]. The fluorescence light profile for the case of vertical incidence can be seen in Fig. 18 and the corresponding difference in Fig. 19. The expected light of the air shower is reduced by $11.1 \%$ during summer, $8.9 \%$ during autumn, $7.3 \%$ during spring, and $6.8 \%$ during winter [71]. For the same shower with $60^{\circ}$ inclination, the expected light is reduced by $8.4 \%$ during winter, $8.1 \%$ during spring and autumn, and $8.0 \%$ during summer. The same calculations have been performed for protoninduced air showers. The reduction of the expected light is increased by about $0.5 \%$ compared to the numbers of the iron-induced cascade [71]. Hence, accounting for the newly measured altitude dependences in the reconstruction of the primary energy of the shower, the primary energy will be increased by this amount as compared with the former model calculations.

\section{Summary and Outlook}

The 5th Fluorescence Workshop has brought together experimental and theoretical expertise from around the world to discuss the status of the determination of the fluorescence light yield of electrons, important for air shower observations. It can be realized with pleasure that over the last years significant progress has been achieved in both, experimental and theoretical work.

Convergence has been achieved in several aspects between the different groups. Most important for air shower observations is the fluorescence light yield of nitrogen, since the contributions of direct excitation of oxygen and argon seem to be negligible. Hence, investigations focus on the fluorescence light emission of nitrogen. The position of the molecular bands in the fluorescence light spectrum is well known. Accurate measurements of the relative intensities at typical laboratory conditions have been presented. Also high precision values of the characteristic pressures have been reported, although full agreement is still missing. High-precision $P^{\prime}$ values allow accurate predictions on the relative fluorescence intensity at any atmospheric condition of interest for air shower reconstruction. The proportionality between fluorescence intensity and deposited energy seems to be proved from both experimental and theoretical sides. Recent investigations reveal that the dependence of the light yield on temperature and humidity can not be neglected. In realistic atmospheres effects up to $10 \%$ can be expected. Further measurements are necessary to clarify the situation.

A precise determination of the absolute light yield is extremely difficult and still needs more experimental data. For a comparison of the values obtained by different groups, it would be useful if the same concept is used. The participants agreed to specify the fluorescence light yield in photons per energy depo- 
sition (photons/MeV). This quantity is most useful to convert the observed light yield into a calorimetric measurement of the energy of an air shower. The usage of this unit is strongly encouraged.

The open questions are presently addressed by several groups. The workshop participants expressed their hope to achieve convergence on the open issues soon $(1-2$ years $)$.

\section{Acknowledgements}

The authors acknowledge the support of the Spanish Ministry of Science and Education MEC (FPA2006-12184-C02-01), Comunidad de Madrid (Ref.: 910600) and CONSOLIDER program, and of the German Research Foundation (DFG) (KE 1151/1-1 and KE 1151/1-2). Further support has been granted by the Radboud Universiteit Nijmegen as well as by the Universität Karlsruhe (TH) and the Forschungszentrum Karlsruhe $\mathrm{GmbH}$ which are currently merging their activities in the Karlsruhe Institute of Technology (KIT). The authors would like to thank C. Escobar, C. Field, M. Fraga, K. Martens, M. Nagano, J. Ridky, J. Rosado, and A. Ulrich for valuable comments on the manuscript.

\section{References}

[1] J. Linsley, Phys. Rev. Lett. 10 (1963) 146.

[2] National Research Council, Connecting quarks with the cosmos - Eleven science questions for the new century. National Academy Press, Washington, D.C. (2003).

[3] M. Nagano, A. Watson, Rev. Mod. Phys. 72 (2000) 689.

[4] D. Bergman, J. Belz, J. Phys. G: Nucl. Part. Phys. 34 (2007) R359.

[5] K.-H. Kampert, arXiv:0801.1986 (2008).

[6] J.R. Hörandel, arXiv:0803.3040 (2008).

[7] F. Arqueros, J.R. Hörandel, B. Keilhauer, Proc. of the 5th Fluorescence Workshop, Nucl. Instr. \& Meth. A (2008) in press.

[8] C.W. Fabjan, F. Gianotti, Rev. Mod. Phys. 75 (2003) 1243.

[9] M. Risse, D. Heck, Astropart. Phys. 20 (2004) 661.

[10] F. Nerling et al., Astropart. Phys. 24 (2006) 421.

[11] T. Pierog et al., in: Proc. 29th Int. Cosmic Ray Conf., Pune, India, 7 (2005) 103.

[12] J.A. Bellido et al., in: Proc. 29th Int. Cosmic Ray Conf., Pune, India, 7 (2005) 13.

[13] B. Keilhauer et al., Astropart. Phys. 22 (2004) 249.

[14] K. Suga, in Proc. 5th Interamerican Seminar on Cosmic Rays, I. Escobar et al. (eds.) 2 (1962) XLIX. and A.E. Chudakov, comment to this contribution.

[15] G. Tanahashi, AIP Conf. Proc. 433, F. Krizmanic et al. (eds.), American Institute of Physics, New York (1977) 54.
[16] A.N. Bunner, Cosmic Ray Detection by Atmospheric Fluorescence, PhD thesis, Cornell University, 1967.

[17] T. Hara et al., Acta Physica Academiae Hungaricae 29 suppl. 3 (1970) 369.

[18] R.M. Baltrusaitis et al., Nucl. Instr. and Meth. A 240 (1985) 410, and 264 (1988) 87.

[19] T. Abu-Zayyad et al., Nucl. Instr. and Meth. A 450 (2000) 253.

[20] M. Fukushima et al., in: Proc. 30th Int. Cosmic Ray Conf., Merida, Mexico (2007) in press.

[21] S. Ogio et al., in: Proc. 30th Int. Cosmic Ray Conf., Merida, Mexico (2007) in press.

[22] J. Abraham et al. (Pierre Auger Coll.), Nucl. Instr. and Meth. A 523 (2004) 50.

[23] H. Klages et al. for the Pierre Auger Coll., in: Proc. 30th Int. Cosmic Ray Conf., Merida, Mexico (2007) in press.

[24] A. Etchegoyen et al. for the Pierre Auger Coll., in: Proc. 30th Int. Cosmic Ray Conf., Merida, Mexico (2007) in press.

[25] A. van den Berg et al. for the Pierre Auger Coll., in: Proc. 30th Int. Cosmic Ray Conf., Merida, Mexico (2007) in press.

[26] D. Nitz et al. for the Pierre Auger Coll., in: Proc. 30th Int. Cosmic Ray Conf., Merida, Mexico (2007) in press (arXiv:0706.3940).

[27] Y. Aita et al., in: Proc. 28th Int. Cosmic Ray Conf., Tsukuba, Japan, 2 (2003) 1061.

[28] Y. Takizawa et al., Nucl. Phys. B (Proc. Suppl.) 166 (2007) 72.

[29] M. Ave et al. (AIRFLY Coll.), Astropart. Phys. 28 (2007) 41.

[30] R.W.B. Pearse and A.G. Gaydon, The identification of molecular spectra, London: Chapman and Hall, New York: John Wiley \& Sons, 1976, 4th ed.

[31] D.J. Eckstrom et al., J. Chem. Phys. 82 (1985) 1792.

[32] M. Ave et al. (AIRFLY Coll.), Proc. of the 5th Fluorescence Workshop, Nucl. Instr. \& Meth. A (2008) in press.

[33] F. Blanco, F. Arqueros, Phys. Lett A 345 (2005) 355.

[34] F. Arqueros et al., Astropart. Phys. 26 (2006) 231.

[35] F.R. Gilmore et al., J. Chem. Ref. Data 21 (1992) 1005.

[36] C.O. Laux, C.H. Kruger, J. Quant. Spectrosc. Radiat. Transfer 48 (1992) 9.

[37] O. Stern, M. Volmer, Physik. Zeitschr. 20 (1919) 183.

[38] A. Morozov et al., Proc. of the 5th Fluorescence Workshop, Nucl. Instr. \& Meth. A (2008) in press.

[39] A. Morozov et al., Eur. Phys. J. D 46 (2008) 51.

[40] J.T. Yardley, Introduction to Molecular Energy Transfer, Academic, New York, (1980).

[41] M.M. Fraga et al., Proc. of the 5th Fluorescence Workshop, Nucl. Instr. \& Meth. A (2008) in press.

[42] D.L. Holtermann et al., J. Chem. Phys. 77 (1982) 5327.

[43] A.E. Bailey et al., J. Chem. Soc., Faraday Trans. 93 (1997) 2915.

[44] M. Ave et al. (AIRFLY Coll.), Proc. of the 5th Fluorescence Workshop, Nucl. Instr. \& Meth. A (2008) in press.

[45] F. Kakimoto et al., Nucl. Instr. and Meth. A372 (1996) 527.

[46] M. Nagano et al., Astropart. Phys. 20 (2003) 293.

[47] M. Nagano et al., Astropart. Phys. 22 (2004) 235.

[48] J.W. Belz et al. (FLASH Coll.), Astropart. Phys. 25 (2006) 129. 
[49] R. Abbasi et al., Astropart. Phys. 29 (2008) 77.

[50] T. Waldenmaier et al., Proc. of the 5th Fluorescence Workshop, Nucl. Instr. \& Meth. A (2008) in press.

[51] P. Hüntemeyer et al. (FLASH Coll.), Proc. of the 5th Fluorescence Workshop, Nucl. Instr. \& Meth. A (2008) in press.

[52] P. Colin et al. (MACFLY Coll.), Astropart. Phys. 27 (2007) 317.

[53] M. Ave et al. (AIRFLY Coll.), Proc. of the 5th Fluorescence Workshop, Nucl. Instr. \& Meth. A (2008) in press.

[54] G. Lefeuvre et al., Nucl. Inst. and Meth. A 578 (2007) 78.

[55] F. Arqueros et al., Proc. of the 5th Fluorescence Workshop, Nucl. Instr. \& Meth. A (2008) in press.

[56] A. Morozov et al., Eur. Phys. J. D33 (2005) 207.

[57] J. Rosado et al., Proc. of the 5th Fluorescence Workshop, Nucl. Instr. \& Meth. A (2008) in press.

[58] J. Belz et al. (FLASH Coll.), Proc. of the 5th Fluorescence Workshop, Nucl. Instr. \& Meth. A (2008) in press.

[59] J. Belz et al. (FLASH Coll.), Astropart. Phys. 25 (2006) 5763.

[60] P. Colin et al. (MACFLY Coll.), submitted to Astropart. Phys. (arXiv:astro-ph/0703230v1).

[61] N. Sakaki et al., Proc. of the 5th Fluorescence Workshop, Nucl. Instr. \& Meth. A (2008) in press.

[62] M. Ave et al. (AIRFLY Coll.), Proc. of the 5th Fluorescence Workshop, Nucl. Instr. \& Meth. A (2008) in press.

[63] W.R. Nelson, H. Hiragama and D.W.O. Rogers, The EGS4 Code System, SLAC-265, Stanford Linear Accelerator Center (1985).

[64] S. Agostinelli, et al., GEANT4: A simulation toolkit, Nucl. Instr. \& Meth. A 506 (2003) 250.

[65] 〈http://lappweb.in2p3.fr/IWFM05/〉.

[66] S.V. Pancheshnyi et al., Chem. Phys. 262 (2000) 349.

[67] S.V. Pancheshnyi et al., Chem. Phys. Lett. 294 (1998) 523.

[68] P. Privitera for the AIRFLY Coll., in: Proc. 30th Int. Cosmic Ray Conf., Merida, Mexico, (2007) in press.

[69] A.E. Grün, E. Schopper, Z. Naturforschg. 9a, (1954) 55.

[70] D.C. Lillicrap, Collision quenching effects in nitrogen and helium excited by a $30-\mathrm{keV}$ electron beam, NASATM-X-2842, (1973).

[71] B. Keilhauer et al., Proc. of the 5th Fluorescence Workshop, Nucl. Instr. \& Meth. A (2008) in press.

[72] N. Sakaki et al., Proc. of the 5th Fluorescence Workshop, Nucl. Instr. \& Meth. A (2008) in press.

[73] T. Waldenmaier et al., arXiv:0709.1494 (2007).

[74] W.R. Bennett Jr., J. Flint, Phys. Rev. A18 (1978) 2527.

[75] A. Morozov et al., Appl. Phys. Lett. 86 (2005) 011502.

[76] R.W. Nicolls et al., Proc. Phys. Soc. 74 (1959) 87.

[77] National Aeronautics and Space Adminstration (NASA), U.S. Standard Atmosphere 1976, NASA-TMX-74335, (1976).

[78] B. Keilhauer et al., Astropart. Phys. 25 (2006) 259.

[79] J. Knapp, D. Heck, Extensive Air Shower Simulation with CORSIKA: A User's Guide, Report KfK 5196B, Kernforschungszentrum Karlsruhe, (1993); for an up-todate version see $\langle$ http://www-ik.fzk.de/corsika $\rangle$. 